Linköping University Medical Dissertations No. 1592

\title{
Assessment of Ventricular Function in Normal and Failing Hearts Using 4D Flow CMR
}

\author{
Jakub Zajac
}

\author{
Division of Cardiovascular Medicine \\ Department of Medical and Health Sciences \\ Faculty of Medicine and Health Sciences \\ Center for Medical Image Science and Visualization \\ Linköping University, Sweden \\ Linköping, September 2017
}


This work has been conducted within the Center for Medical Image Science and Visualization (CMIV) at Linköping University, Sweden. The Swedish Heart-Lung foundation, the Swedish Research Council, the Emil and Wera Cornell Foundation and the European Research Council are acknowledged for financial support.

Assessment of Ventricular Function in Normal and Failing Hearts Using 4D Flow CMR

Linköping University Medical Dissertations No. 1592

Copyright (C) Jakub Zajac 2017

No part of this work may be reproduced, stored in a retrieval system, or be transmitted in any form or by any means, electronic, mechanic, photocopying, recording or otherwise, without prior written permission from the author.

Division of Cardiovascular Medicine

Department of Medical and Health Sciences

Linköping University

SE-581 85 Linköping, Sweden

http://www.liu.se/cmr

ISBN: 978-91-7685-438-9

ISSN 0345-0082

Printed by LiU-Tryck, Linköping, Sweden, 2017

Front cover: Multicolor drawing of the heart and the outflow by Dr. Staffan Wirell, handed to me the day I became a PhD student. Permission to print. 


\section{"I've got an elastic heart Yeah, I've got an elastic heart" \\ - Sia}





\section{ABSTRACT}

Heart failure is a common disorder and a major cause of illness and death in the population, creating an enormous health-care burden. It is a complex condition, representing the end-point of many cardiovascular diseases. In general heart failure progresses slowly over time and once it is diagnosed it has a poor prognosis which is comparable with that of many types of cancer.

The heart has an ability to adapt in response to long lasting increases in hemodynamic demand; the heart conforms its shape and size in order to maintain adequate cardiac output. This process is called remodeling and can be triggered by pathologies such as hypertension or valvular disease. When the myocardial remodeling is maintained chronically it becomes maladaptive and is associated with an increased risk of heart failure.

In many cases, heart failure is associated with left bundle branch block (LBBB). This electrical disturbance leads to dyssynchronous left ventricular (LV) contraction and relaxation which may contribute to cardiac dysfunction and ultimately heart failure. Mechanical dyssynchrony can be treated with cardiac resynchronization therapy (CRT). However, many heart failure patients do not demonstrate clinical improvement despite CRT.

Blood flow plays an important role in the normal development of the fetal heart. However, flow-induced forces may also induce changes in the heart cells that could lead to pathological remodeling in the adult heart. Until recently, measurement tools have been inadequate in describing the complex three-dimensional and time-varying characteristics of blood flow within the beating heart.

4D (3D + time) flow cardiovascular magnetic resonance (CMR) enables acquisition of three-dimensional, three-directional, time-resolved velocity data from which visualization and quantification of the blood flow patterns over a complete cardiac cycle can be performed. In this thesis, novel 4D Flow CMR based methods are used to study the intraventricular blood flow in healthy subjects and heart failure patients with and without ventricular dyssynchrony in order to gain new knowledge of the ventricular function.

Different flow components were assessed in normal heart ventricles. It was found that inflowing blood that passes directly to outflow during the same heartbeat (the Direct Flow component) was larger and possessed more 
kinetic energy (KE) than other flow components. Diastolic flow through the normal heart appears to create favorable conditions for effective systolic ejection. This organized blood flow pattern within the normal LV is altered in heart failure patients and is associated with decreased preservation of KE which might be unfavorable for efficient LV ejection. Inefficient flow of blood through the heart may influence diastolic wall stress, and thus contribute to pathological myocardial remodeling.

In dyssynchronous LVs of heart failure patients with LBBB, Direct Flow showed even more reduced preservation of KE compared to similarly remodeled LVs without LBBB. Furthermore, in LBBB patients, LV filling hemodynamic forces, acting on the myocardium, were more orthogonal to the main flow direction compared to patients without LBBB. Deviation of LV flow forces and reduction of KE preservation and may reflect impairment of LV diastolic function and less efficient ensuing ejection related to dyssynchrony in these failing ventricles.

Blood flow patterns were also studied with respect to fluctuations of the velocity of the flow (turbulent flow) in normal and failing LVs. In failing hearts, turbulent kinetic energy (TKE) was higher during diastole than in healthy subjects. TKE is a cause of energy loss and can thus be seen as a measure of flow inefficiency.

Elucidating the transit of multidimensional blood flow through the heart chambers is fundamental in understanding the physiology of the heart and to detect abnormalities in cardiac function. The 4D Flow CMR parameters presented in this thesis can be utilized to detect altered intracardiac blood flow and may be used as markers of deteriorating cardiac function, pathological remodeling and mechanical dyssynchrony in heart failure. 


\section{POPULÄRVETENSKAPLIG SAMMANFATTNING}

Hjärtsvikt är ett vanligt tillstånd och utgör en stor sjukvårdsbörda. Den representerar slutstadiet av våra hjärt-kärlsjukdomar och beror på att hjärtat inte förmår att pumpa runt tillräckligt med blod i kroppen. Ofta diagnostiseras hjärtsvikt sent i sjukdomsförloppet. Vid manifesta symptom är hjärtsvikten långt framskriden och har en dålig prognos som kan jämföras med prognosen för flera vanliga cancersjukdomar.

Hjärtsvikt utvecklas ofta sakta och utan symptom under relativt lång tid vilket beror på att hjärtat ändrar sin storlek och form med tiden för att upprätthålla adekvat blodförsörjning, ett fenomen som kallas hjärtremodellering. Remodellering kan ske som svar på sjukliga tillstånd som till exempel högt blodtryck eller sjukdomar i hjärtklaffarna. Detta är dock ogynnsamt för hjärtat på längre sikt.

Det är känt att samspelet mellan blodflödesmönster och hjärtmuskel har betydelse för hjärtats normala utveckling under fostertiden. Krafter skapade av blodflödet kan dock även bidra till skadlig remodellering av det vuxna hjärtat. Kartläggning av blodflödets invecklade vägar genom hjärtat är grundläggande för att förstå hjärtats funktion och sjukdomar. Tidigare metoder har varit begränsade i sin förmåga att mäta det tre-dimensionella blodflödet. Fyrdimensionellt (tre dimensioner + tidsdimension) flöde med hjälp av magnetkameraundersökning av hjärtat möjliggör visualisering och kvantifiering av det komplexa blodflödet genom hjärtat.

Blodflöden inuti hjärtat kan med nya "4D Flow CMR" metoder delas upp i olika komponenter utifrån blodets flödesvägar genom kammaren och dess rörelseenergi beräknas. De kan även studeras utifrån hur flödeshastighet varierar (turbulent blodflöde). Dessutom kan man beräkna den kraft som blodflöde utövar på hjärtmuskelväggarna (hemodynamisk kraft). Turbulent blodflöde står ofta för förlust av energi och hemodynamisk kraft kan öka belastningen i hjärtmuskeln och trigga skadlig remodellering.

Syftet med avhandlingen är att med dessa nya blodflödesspecifika mått öka förståelsen för hjärtats normala funktion samt hur hjärtsvikt och vänstersidigt skänkelblock påverkar funktionen.

Hos friska individer har det observerats att merparten av blodflödet genom hjärtkamrarna utgörs av det som kommer in och pumpas ut från kamrarna under ett och samma hjärtslag. Detta "direktflöde" kan avspegla en effektiv koppling mellan hjärtats vilofas och arbetsfas och god pumpeffektivitet. 
Det välorganiserade flödesmönstret $\mathrm{i}$ normala hjärtan är rubbat hos patienter med hjärtsjukdom - volymen av direktflödet är mindre och dess rörelseenergi är lägre. Dessutom finns högre grad av turbulent flöde. Dessa fynd kan vara uttryck för sämre pumpeffektivitet. Vidare har ännu lägre nivåer av rörelseenergi hos direktflödet $i$ vänster hjärtkammare iakttagits $i$ sviktande hjärtan med försenad elektrisk aktivering av vänster kammare jämfört med sviktande hjärtan utan denna försening. Hos de med försenad aktivering var även blodflödets hemodynamiska kraft i större utsträckning riktad vinkelrätt mot blodflödets normala rörelseriktning. Dessa aspekter kan förklara en försämrad funktion i hjärtats vilofas som är förknippad med icke samtidig vänster- och högerkammarpumpning.

Förändrat blodflödesmönster inuti hjärtat kan vara en bakomliggande mekanism och således markör för tilltagande hjärt-remodellering. Ökad kunskap om sambandet mellan förändrat blodflöde i hjärtat och remodellering hos patienter med hjärtsvikt kan bidra med viktig information vid diagnostisering och uppföljning av behandling. Identifiering av riskpatienter kan bidra till optimerad behandling av dessa patienter, vilket på sikt kan leda till minskade sjukvårdskostnader. 


\section{LIST OF PAPERS}

This thesis is based on the following papers:

I 4-D blood flow in the human right ventricle.

Alexandru G. Fredriksson*, Jakub Zajac*, Jonatan Eriksson, Petter Dyverfeldt, Ann F. Bolger, Tino Ebbers, Carl-Johan Carlhäll. American Journal of Physiology - Heart and Circulatory Physiology. 2011;301:H2344-50.

* Contributed equally to the paper.

II Turbulent kinetic energy in normal and myopathic left ventricles.

Jakub Zajac, Jonatan Eriksson, Petter Dyverfeldt, Ann F. Bolger, Tino Ebbers, Carl-Johan Carlhäll.

Journal of Magnetic Resonance Imaging. 2015;41:1021-9.

III Mechanical dyssynchrony alters left ventricular flow energetics in failing hearts with LBBB.

Jakub Zajac, Jonatan Eriksson, Urban Alehagen, Tino Ebbers, Ann F. Bolger, Carl-Johan Carlhäll.

Submitted manuscript.

IV Left ventricular hemodynamic forces as a marker of mechanical dyssynchrony in heart failure patients with left bundle branch block.

Jonatan Eriksson, Jakub Zajac, Urban Alehagen, Ann F. Bolger, Tino Ebbers, Carl-Johan Carlhäll.

Scientific Reports. 2017;7:2971. 



\section{ABBREVIATIONS}

$\begin{array}{ll}{ }^{1} \mathrm{H} & \text { hydrogen atom } \\ \text { 2ch } & \text { two-chamber view } \\ \text { 2D } & \text { two-dimensional } \\ \text { 3ch } & \text { three-chamber view } \\ \text { 3T } & \text { three Tesla } \\ \text { 4ch } & \text { four-chamber view } \\ \text { 4D } & \text { four-dimensional } \\ \text { AoV } & \text { aortic valve } \\ \text { ATP } & \text { adenosine triphosphate } \\ \text { AV } & \text { atrioventricular } \\ \text { bSSFP } & \text { balanced steady-state free precession } \\ \text { Ca }{ }^{2+} & \text { calcium ion } \\ \text { CFD } & \text { computational fluid dynamics } \\ \text { CMR } & \text { cardiovascular magnetic resonance } \\ \text { CRT } & \text { cardiac resynchronization therapy } \\ \text { CT } & \text { computed tomography } \\ \text { DCM } & \text { dilated cardiomyopathy } \\ \text { ECG } & \text { electrocardiography } \\ \text { echo-PIV } & \text { echocardiographic particle image velocimetry } \\ \text { ED } & \text { end-diastole } \\ \text { EDV } & \text { end-diastolic volume } \\ \text { EF } & \text { ejection fraction } \\ \text { ES } & \text { end-systole } \\ \text { ESV } & \text { end-systolic volume } \\ \text { FID } & \text { free-induction decay } \\ \text { GRE } & \text { gradient-echo } \\ \text { ICM } & \text { ischemic cardiomyopathy } \\ \text { IVC } & \text { isovolumetric contraction } \\ \text { IVR } & \text { isovolumetric relaxation } \\ \text { KE } & \text { kinetic energy } \\ \text { LA } & \text { left atrium } \\ \text { LAx } & \text { long-axis } \\ \text { LBBB } & \text { left bundle branch block } \\ \text { LV } & \text { left ventricle } \\ \text { LVEDV } & \text { left ventricular end-diastolic volume } \\ \text { LVOT } & \text { left ventricular outflow tract } \\ \text { mJ } & \text { milliJoule } \\ \text { MR } & \text { magnetic resonance } \\ \text { MRI } & \text { magnetic resonance imaging } \\ \text { ms } & \text { millisecond } \\ & \end{array}$




$\begin{array}{ll}\text { MV } & \text { mitral valve } \\ \text { QRS } & \text { Q, R and S wave on ECG } \\ \text { RA } & \text { right atrium } \\ \text { RBBB } & \text { right bundle branch block } \\ \text { RF } & \text { radio frequency } \\ \text { RV } & \text { right ventricle } \\ \text { SA } & \text { sinoatrial } \\ \text { SAx } & \text { short-axis } \\ \text { SE } & \text { spin-echo } \\ \text { SENSE } & \text { sensitivity encoding } \\ \text { SNR } & \text { signal-to-noise ratio } \\ \text { SR } & \text { sarcoplasmic reticulum } \\ \text { TE } & \text { echo time } \\ \text { TKE } & \text { turbulent kinetic energy } \\ \text { TnC } & \text { troponin C } \\ \text { TR } & \text { repetition time } \\ \text { VENC } & \text { velocity encoding }\end{array}$




\section{TABLE OF CONTENTS}

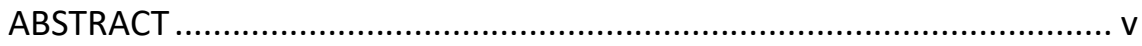

POPULÄRVETENSKAPLIG SAMMANFATTNING ......................................vii

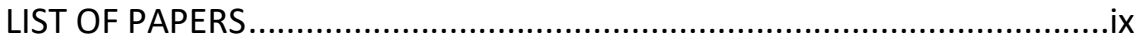

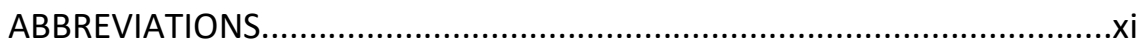

TABLE OF CONTENTS .....................................................................

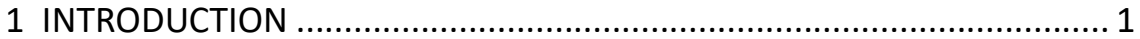

2 AIMS

3 PHYSIOLOGY OF THE HEART …………………............................... 5

3.1 Cardiac action potential ........................................................... 5

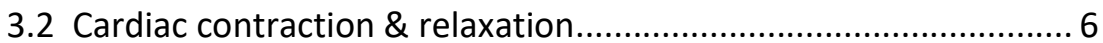

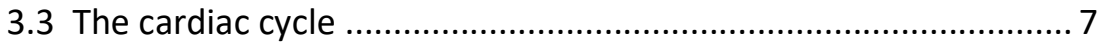

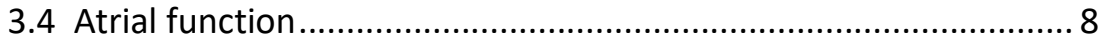

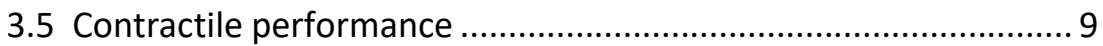

3.6 Myocardial fiber orientation ..................................................... 10

3.7 Interventricular interaction ......................................................... 10

4 HEART FAILURE \& CARDIAC REMODELING....................................... 13

4.1 Neurohormonal adaptation ...................................................... 13

4.2 Hypertrophy \& remodeling ...................................................... 14

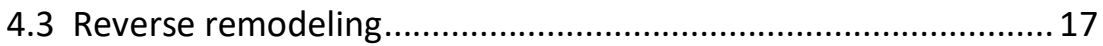

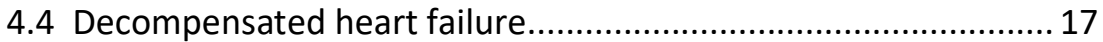

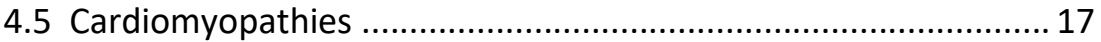

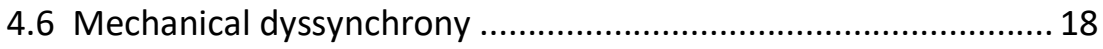

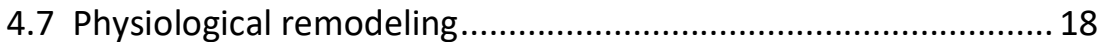

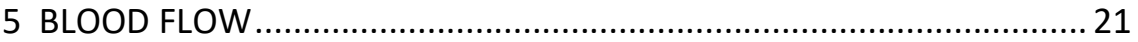

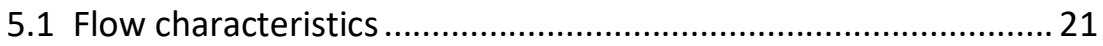

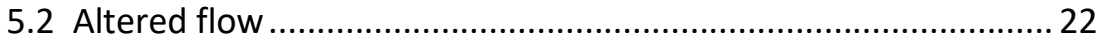




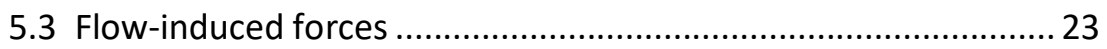

6 CARDIAC IMAGING \& ASSESSMENT OF CARDIAC FUNCTION ............. 25

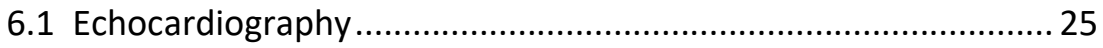

6.2 Cardiovascular magnetic resonance imaging ............................. 26

6.3 Assessment of cardiac function .................................................. 26

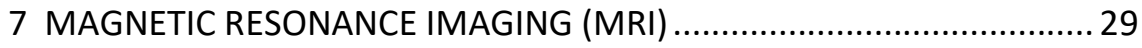

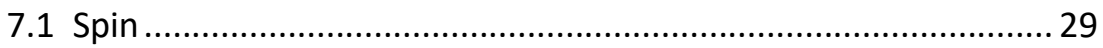

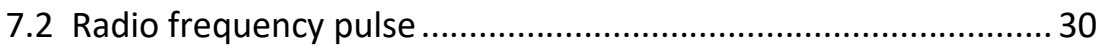

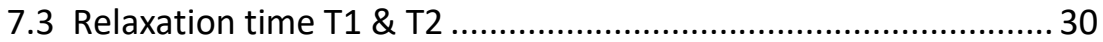

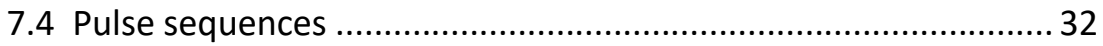

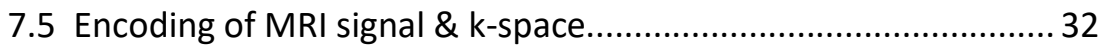

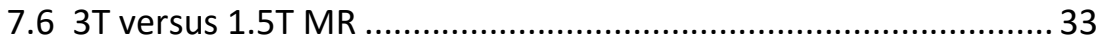

8 CARDIOVASCULAR MAGNETIC RESONANCE IMAGING (CMR) ............ 35

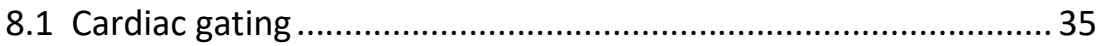

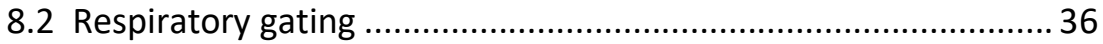

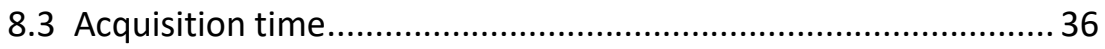

8.4 Balanced steady-state free-precession ....................................... 36

9 4D FLOW CMR

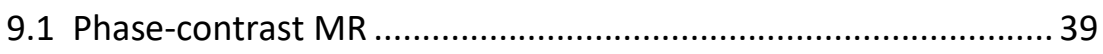

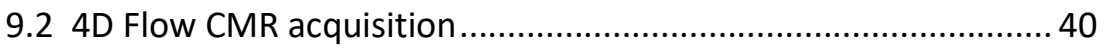

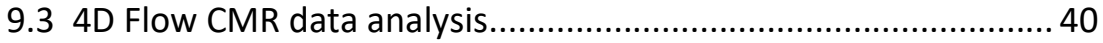

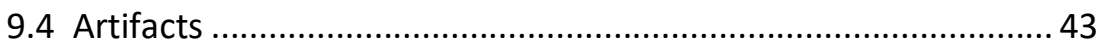

10 4D FLOW CMR ANALYSES \& PRESENT STUDIES................................. 45

10.1 Flow analysis in normal right ventricle (paper I) ........................ 46

10.2 TKE in normal \& myopathic left ventricles (paper II) ................ 48

10.3 Flow analysis in failing hearts with \& without LBBB (paper III) 48

10.4 Hemodynamic force in failing hearts with \& without LBBB

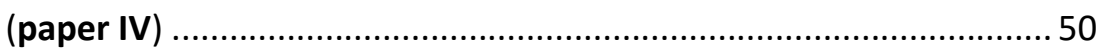




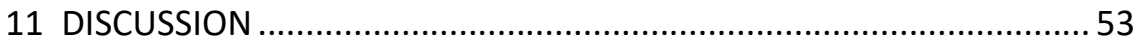

11.1 Physiological considerations ................................................... 53

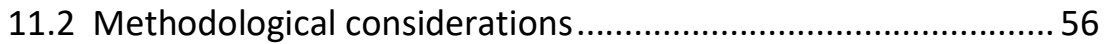

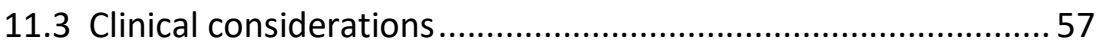

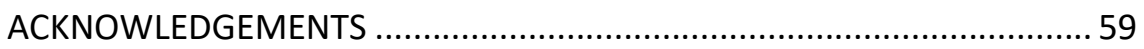

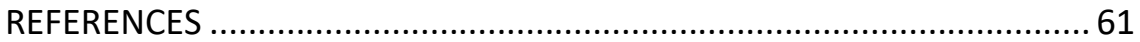





\section{INTRODUCTION}

Heart failure is a serious and deadly condition. It is relatively common, in part due to the aging of the population and in part due to better treatment of underlying cardiac diseases and higher survival rate (1). It is in about $1 \%$ of cases the primary reason for emergency hospital admissions (2). Prognosis of heart failure is poor, even worse than many types of cancer (3). $30-40 \%$ of heart failure patients die within a year of diagnosis and 60 $70 \%$ within 5 years (4). It is also disabling and reduces quality of life (5).

Heart failure cannot be looked on as a simple condition of abnormal heart pumping or a disease of the heart alone (6). In fact, heart failure is an extremely complex disease, representing the end-stage of many cardiovascular and also non-cardiovascular diseases. Heart failure in itself is a symptom of failure of the heart to pump adequate amount of blood to the body.

There are common features of heart failure such as increase in heart size, deterioration of cardiac function and decompensation with symptoms of inadequate cardiac output. Current theories for explaining the pathophysiology of heart failure are not able to adequately explain and predict disease progression (1). As a result, these models do not provide adequate frameworks for understanding new treatment strategies. A deeper knowledge of heart failure pathophysiology would add to future therapeutic advances.

It is known that flow-induced forces can alter the shape of the heart (7). How these contribute to heart failure progression is not clarified. Previously, measurement tools have been inadequate in describing the complex three-dimensional and time-varying characteristics of blood flow within the beating heart. Intraventricular flow patterns and characteristics have in recent years been visualized and quantified using 4D Flow CMR. This novel and promising method has provided new insight into the complicated hemodynamics of normal and failing hearts. 


\section{AIMS}

The overall aim of this doctoral work was to study intraventricular blood flow by applying 4D Flow CMR based methods in healthy subjects and heart failure patients with and without ventricular dyssynchrony, in order to gain novel aspects of ventricular function.

The specific aims of the papers included in this thesis were as follows:

\section{Paper I}

To characterize the blood flow through the right ventricle of the heart and compare to that of the left ventricle.

\section{Paper II}

To assess turbulent kinetic energy within the left ventricle of healthy subjects and compare it to those of patients with dilated cardiomyopathy.

\section{Paper III}

To investigate blood flow patterns and kinetic energy in myopathic left ventricles with and without left bundle branch block.

\section{Paper IV}

To assess hemodynamic forces in failing left ventricles with and without left bundle branch block. 


\section{PHYSIOLOGY OF THE HEART}

Cardiac muscle cells (myocytes) constitute approximately $75 \%$ of the total volume of the myocardium and contain the contractile elements, myofibrils. The normal heart contractility depends on key cell structures that comprises the myofilaments, actin and myosin, of the myofibrils that are arranged into sarcomeres. The sarcoplasmic reticulum (SR) is crucial for storing calcium $\left(\mathrm{Ca}^{2+}\right)$ and for initiating contraction. The myocyte is confined by a cell membrane, called sarcolemma, which forms tubular tunneled networks ( $\mathrm{T}$ tubules). Mitochondria in the muscle cells are essential for the production of adenosine triphosphate (ATP), the main form of energy for generating cardiac contraction as well as for maintaining ion gradient with the help of energy-dependent ion channels.

\subsection{Cardiac action potential}

In the sinoatrial node (SA node) of the right atrium (RA), the pacemaker cells initiate an electric impulse, the action potential, which spreads though the cardiac conductive system and generates a contraction (Fig.1). At the atrioventricular node (AV node), the impulse is delayed to allow the ventricles to finish filling with blood (8). After that, the impulse spreads through the bundle of His and then via right and left bundle branches.

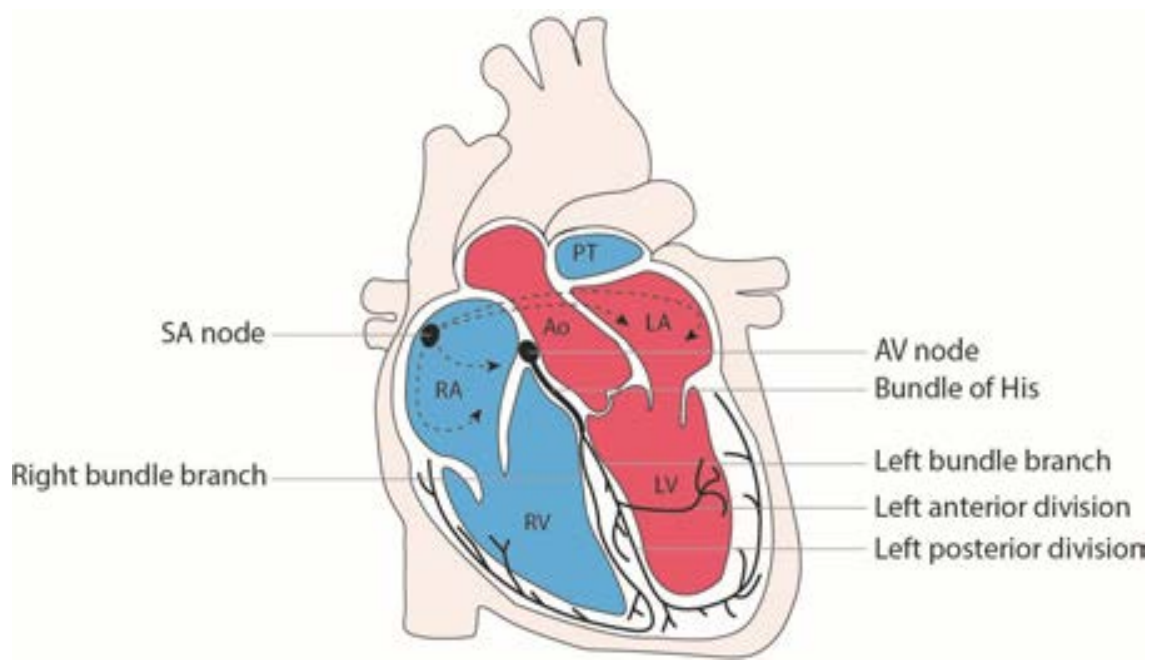

Figure 1: Cardiac conduction system. Ao, aorta; LA, left atrium; LV, left ventricle; PT, pulmonary trunk; RA, right atrium; RV, right ventricle. 
The left bundle branch divides into an anterior and posterior fascicle. Lastly Purkinje fibers propagate the action potential in the walls of the ventricles from cell to cell through gap junctions. In normal conditions, the SA node controls the duration of the cardiac cycle, while the cardiac conduction system and myocytes controls the duration of contraction and relaxation.

In some pathological conditions, the right or left bundle branches may be damaged and unable to effectively propagate the impulse further towards the ventricles. This leads to a delayed contraction in the ventricle of the blocked bundle branch.

\subsection{Cardiac contraction \& relaxation}

When the action potential reaches the $\mathrm{T}$ tubules, voltage-gated $\mathrm{Ca}^{2+}$ channels open leading to entry of $\mathrm{Ca}^{2+}$, which in turn triggers a much larger release of $\mathrm{Ca}^{2+}$ from the SR. This high influx of $\mathrm{Ca}^{2+}$ initiates myocardial contraction. At rest complexes called tropomyosin lie along the actin molecules and block active binding sites to myosin. Upon $\mathrm{Ca}^{2+}$ release, $\mathrm{Ca}^{2+}$ binds to a regulatory element called troponin $\mathrm{C}(\mathrm{TnC})$ which form a complex with other troponins that moves tropomyosin and uncovers sites to where myosin can attach. This initiates the actin-myosin interaction, a so called cross-bridging. By consuming one molecule of ATP, the myosin pulls on actin (power stroke) to the center of the sarcomere, shortening the sarcomere, which results in a mechanical force of contraction (Fig.2).

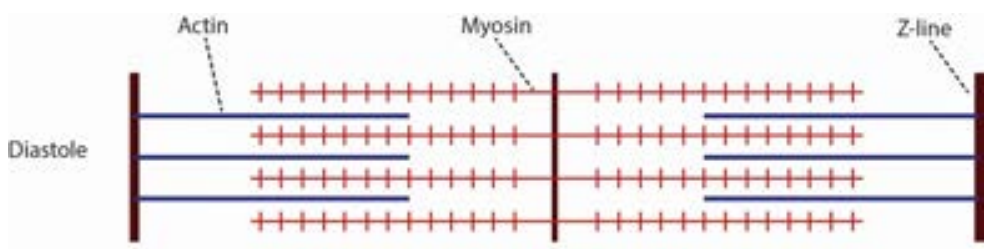

Contraction

Systole

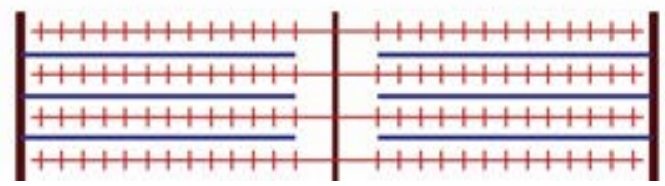

Figure 2: Actin-myosin interaction during diastole and systole. 
Repetitive interaction between myosin and actin (cross-bridge cycle) in many sarcomeres in many myocytes leads to a cardiac contraction. The cascade of biological events that begin with a cardiac action potential and ends with myocyte contraction and relaxation is called excitationcontraction coupling.

The contraction is limited by feedback mechanisms: high concentration of $\mathrm{Ca}^{2+}$ leads to inactivation of $\mathrm{Ca}^{2+}$ channels, uptake of $\mathrm{Ca}^{2+}$ by $\mathrm{SR}$ and decreased $\mathrm{Ca}^{2+}$ interaction with $\mathrm{TnC}$ causes tropomyosin to block actin binding sites and effectively halt power strokes. This leads to relaxation.

\subsection{The cardiac cycle}

The left ventricular (LV) contraction starts shortly after upstroke of the ventricular action potential as $\mathrm{Ca}^{2+}$ binds to and triggers actin-myosin interaction. LV pressure starts building up and when it exceeds that of the left atrium (LA), which normally is around $10-15 \mathrm{mmHg}$, the mitral valve (MV) closes (Fig.3). MV closure along with the closure of tricuspid valve constitute the first heart sound audible with a stethoscope. After this and before the opening of the aortic valve (AoV) the LV volume does not change but pressure increases steadily (isovolumetric contraction, IVC) as more and more myofibers are activated and contract. As the LV pressure rise above the pressure in the aorta, the AoV opens and rapid ejection of blood from LV to aorta ensues. LV pressure rises to a peak and then starts to fall as $\mathrm{SR}$ removes $\mathrm{Ca}^{2+}$, halting the cross-bridging cycles. When the LV pressure falls below the aortic, the AoV closes, producing the second heart sound together with the closure of the pulmonary valve. The LV continues to relax and the pressure continues to decrease, but without any volume change (isovolumetric relaxation, IVR).

When the pressure falls below that in the LA, the MV opens and the LV starts to rapidly fill with blood from the LA, sometimes producing a third heart sound. This phase, called early filling, is caused by pressure difference between the LA and the LV and also by active relaxation of the LV. When these pressures have equalized, roughly halfway through diastole, filling is minimal (diastasis). Atrial contraction, or late filling, thereafter contributes to an additional amount of blood to the LV before the heart cycle starts again. These events are analogous for the right ventricle (RV) albeit at much lower pressure levels. 
The cardiac cycle can be divided into sequential phases in several ways. When considering the heart chambers there are four distinct phases (Fig.3): IVC (point B to C), outflow phase (C to D), IVR (D to A) and inflow phase (A to B). Cardiac systole consists of the first two phases, when the heart is contracting, and diastole of the last two, when the heart is relaxing. The time point between systole and diastole is called end-systole (ES) and between diastole and systole end-diastole (ED).

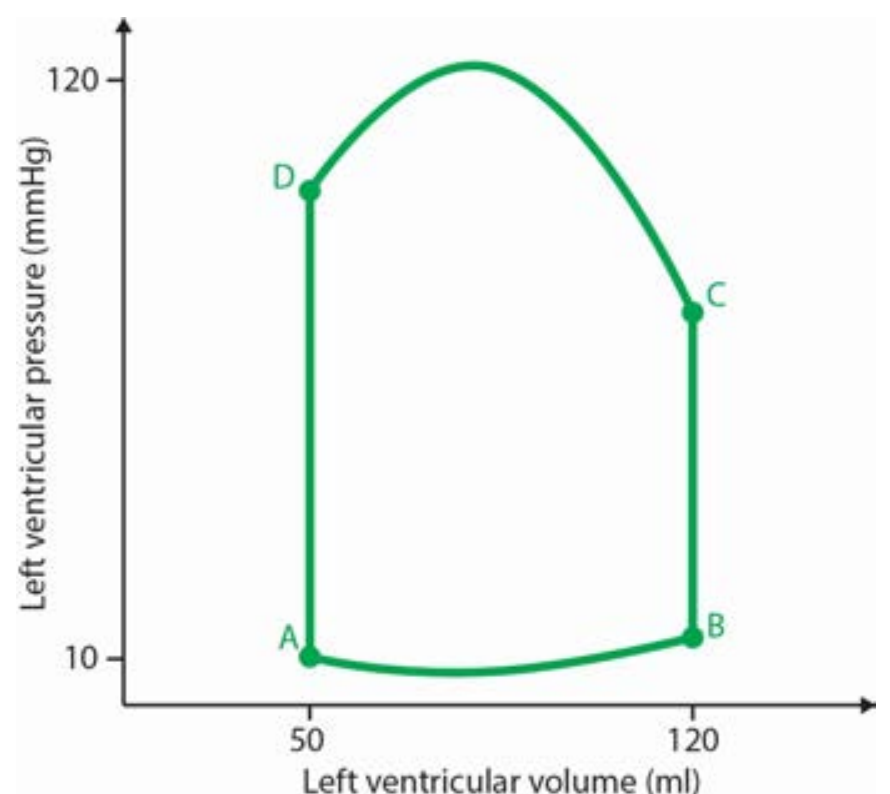

Figure 3: Pressure-volume loop representing events in the cardiac cycle. A) opening of MV. B) closure of MV. C) opening of AoV. D) closure of AoV.

\subsection{Atrial function}

During exercise or other stressful situations which raises the heart rate, the diastolic phase shortens relatively more than systolic (8). As the diastolic phase decreases, less blood will have time to passively enter the LV. Therefore, the rate of LV filling must increase to maintain the LV stroke volume. This is accomplished by faster LV relaxation and lower early diastolic LV pressure, which in part is an effect of enhanced elastic recoil of the myocardium (9). 
At rest, the contribution of the atrial contraction is relatively low and the absence of this contraction, as in atrial fibrillation, would in most cases cause no symptoms. With age and in some myocardial diseases, the heart muscle loses its elasticity. LV relaxation is slower and this leads to a smaller LV-LA pressure difference and reduced blood flow during early diastole. In these cases, atrial contraction contributes more to the LV inflow. Measurements using magnetic resonance imaging have shown that atrial contraction contributes more to the LV filling in older healthy subjects $(38 \%)$ than in younger healthy subjects $(15 \%)(10)$. There was a correlation between atrial contraction contribution and age because of abnormal LV relaxation.

\subsection{Contractile performance}

The performance of the heart depends on several factors of which preload and afterload are extrinsic to the heart. Preload is the degree of filling or load before contraction and it determines the pre-systolic sarcomere length. It can be measured as the end-diastolic volume (EDV), i.e. the volume of blood in the ventricle before contraction. According to Frank-Starling law, the stroke volume of the heart increases in response to an increase in EDV (1). Stroke volume is the volume of blood that is ejected from the LV during systole. If EDV increases (i.e. preload increases), the LV distends more, sarcomere length and force of contraction increases, and stroke volume rises. This can be explained by increased myofilament $\mathrm{Ca}^{2+}$ sensitivity and elevated $\mathrm{Ca}^{2+}$ entry through stretch-activated $\mathrm{Ca}^{2+}$ channels. Afterload is the resistance or load against which the ventricle must pump as it ejects blood during systole. Conditions that increase afterload include aortic stenosis and systemic hypertension. An increase in arterial pressure increases afterload and decreases the stroke volume. Preload and afterload can be more precisely defined with wall stress. The wall stress at ED when the sarcomeres are at the maximum resting length is preload. Afterload is the wall stress during LV ejection.

The intrinsic contractile performance of the heart, independent of the loading conditions, is called contractility or inotropy. Increased or positive inotropic state leads to faster rate of contraction and may be the result of enhanced $\mathrm{Ca}^{2+}$ release/uptake or increased $\mathrm{Ca}^{2+}$ sensitivity of the myofilament (1). Positive inotropy is influenced by adrenergic stimulation, exercise or inotropic medication such as dobutamine. It is often associated with a faster relaxation rate of the myocardium, called lusitropy. 
Heart rate is an important factor for the performance of the heart. Increased heart rate leads to higher cardiac output as it is a product of stroke volume and heart rate. Increased heart rate also leads to enhanced force of contraction due to an increased amount of $\mathrm{Ca}^{2+}$ in the myocytes (1).

The work done by the heart with each heart beat is in part external work which is made up of ejecting a volume of blood against the systolic pressure and delivering kinetic energy (KE) to the ejected blood. The external work is a small part of the total work. Most of the work is internal and consists of maintaining active tension when the heart is not contracting (8). This energy ends up as heat.

\subsection{Myocardial fiber orientation}

The myocardial fibers are oriented in an organized manner through the myocardial wall. This fiber orientation plays an important role in the cardiac pump function. Transmurally through the LV wall, the orientation of fibers are spiral in the subepicardium, circumferential in the middle and longitudinal in the subendocardium (11). The middle layer is the thickest. The myocardial fiber architecture in the RV is comparable to that in the LV except for the middle circumferential layer (12).

In the LV, circular muscle layer constriction resembles a squeezing motion and reduces the intraventricular lumen. The apical portion contracts before the basal parts in order to propel blood upward to the aorta. The spiral and longitudinal muscles are responsible for pulling the mitral annulus towards the apex and thereby shortening the LV long axis (8). The RV empties through three motions: (I) spiral and longitudinal muscles pull the tricuspid annulus towards the apex, (II) the RV free wall moves towards the septum and (III) the contraction of LV circular muscles bulges the septum into the RV lumen. Disarrangement of myocardial fibers may occur in cardiac pathologies such as in cardiomyopathies or fibrosis $(8,13)$.

\subsection{Interventricular interaction}

The LV and RV are anatomically connected within the pericardium (a fluid filled sack that surrounds the heart) and they do not act independent of each other. Muscle fibers from both free ventricles contribute to the interventricular septum. Mechanical coupling between the ventricles has 
been demonstrated in both diastole and systole $(13,14)$. During systole, LV contraction contributes to RV pressure generation through the bulging of the septum into the RV cavity and by transmission of tension to the RV free wall (15). In certain conditions, the RV inflow and end-diastolic pressure rises and can cause the interventricular septum to bulge into the LV cavity and reduce its inflow. 


\section{HEART FAILURE \& CARDIAC REMODELING}

The progression of heart failure is preceded by an event or condition in which the myocardium is unable to pump normally (16). Myocardial infarction is an abrupt event leading to damages in the heart muscle and loss of functioning myocytes, which can initiate the development of heart failure. The initiation can also be gradual as in case of hemodynamic pressure or volume overload. Many heart diseases are hereditary and may trigger heart failure. However, the patient often remains asymptomatic, which is attributed to activation of compensatory mechanisms in response to an initial decline in pumping capacity of the heart.

\subsection{Neurohormonal adaptation}

One of the most important series of compensatory adaptations that maintain homeostasis and cardiac output are collectively known as the neurohormonal model (6). This include activation of the sympathetic (adrenergic) nervous system and renin-angiotensin system. In heart failure, the normal sympathetic inhibitory input from vascular receptors (baroreceptors) located in the carotid sinuses and the aortic arch are depressed due to less stretch as systolic and pulse pressure diminish (17). Instead the sympathetic excitatory input is increased, leading to an overall increase in the sympathetic tone and elevated levels of norepinephrine. Also, at the same time, parasympathetic tone is diminished. Sympathetic activation of beta ${ }_{1}$-adrenergic receptors leads to an increase in heart rate and force of myocardial contraction, both resulting in an increase in cardiac output. Furthermore, increased stimulation of alpha $a_{1}$-adrenergic receptors maintains adequate blood pressure by peripheral vasoconstriction.

In heart failure, renin release from the juxtaglomerular apparatus in the kidneys is increased as a result of renal hypoperfusion, decreased filtered sodium reaching the macula densa in the distal tubule and increased sympathetic stimulation of the kidney. Increase in renin give rise to higher circulating levels of angiotensin II. Angiotensin II acts mainly as a potent vasoconstrictor which is vital in short-term circulatory homeostasis, however, sustained high levels of angiotensin II are maladaptive and lead to fibrosis of the heart (18). Angiotensin II in turn elevates the level of aldosterone secretion by adrenal cortex. Continuously high blood levels of aldosterone may induce hypertrophy and fibrosis within the myocardium, contributing to increased ventricular stiffness (19). Both angiotensin II and 
aldosterone facilitate water and sodium retention in the kidneys, increasing circulatory blood volume as a result. Counter-regulatory mechanisms balance out the effect of these vasoconstrictors. Atrial natriuretic peptide and brain natriuretic peptide are released in response to increased atrial and myocardial stretch and they unload the heart by increasing excretion of water and sodium. In advanced heart failure however, the effect of these peptides is diminished (20).

Several other biologically active molecules are recruited in the compensatory adaptation response, including inflammatory mediators that are responsible for cardiac repair and cardiac remodeling. Elevated force load on the heart puts pressure on the metabolic rate in the heart with increased amount of oxidative stress and higher levels of reactive oxidative species (ROS). ROS can cause numerous deleterious end-effects in myocytes and in the interstitium. Also, the synthesis of the free radical gas nitric oxide have short-term effects on myocyte function and energetics but may have long-term effect on pathological cardiac remodeling (21).

\subsection{Hypertrophy \& remodeling}

Although the neurohormonal model can maintain cardiovascular homeostasis and adequate cardiac output, in general, heart failure progresses slowly over time, transitioning from asymptomatic to symptomatic heart failure. This cannot only be explained by the deleterious effects of the neurohormones. The progression has been attributed to the process of LV remodeling, which is influenced by hemodynamic, neurohormonal, epigenetic and genetic factors. Cardiac remodeling refers to the changes in size, geometry, structure and function of the heart.

With time the failing heart grows in size, a process called hypertrophy. In general, two basic patterns of cardiac hypertrophy ensue in response to hemodynamic overload: concentric and eccentric hypertrophy (1, 22-24). Both patterns of cardiac hypertrophy have been linked to specific cellular signaling pathways (1).

Concentric hypertrophy occurs many times in the presence of pressure overload, for example aortic stenosis or arterial hypertension. In this condition the systolic wall stress is elevated, which leads to an increase of sarcomeres in parallel and in myocyte cross-sectional area (Fig.4). LV wall thickness increases to compensate the increased pressure state. In volume overload state, such as aortic or mitral regurgitation, diastolic wall stress is 
instead increased which leads to an increase in myocyte length with the addition of sarcomeres in series. This increased LV dilation is called eccentric hypertrophy.

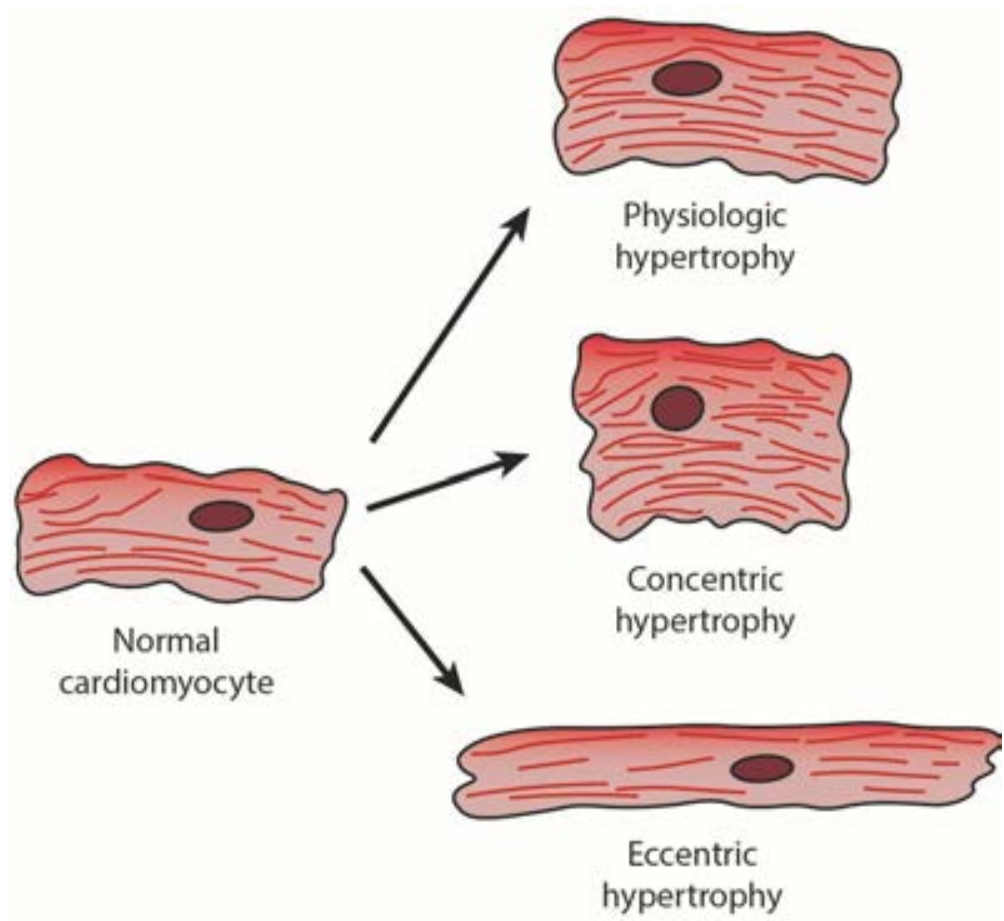

Figure 4: Patterns of cardiac hypertrophy in physiologic and pathological remodeling.

In cardiac hypertrophy, neurohormones, inflammatory cytokines and other peptides and growth factors can reactivate groups of genes that are not expressed postnatally, called fetal gene program, through many intracellular signaling pathways $(1,6,25)$. This is accompanied by a decreased activation of genes that are normally expressed in the adult heart. Mechanical stretch of the myocyte can also reactivate the fetal gene program which may contribute to the contractile dysfunction in the failing myocyte.

In the early stages of hypertrophy, the myocytes are enlarged, with increased number of myofibrils and mitochondria, but they still have 
preserved cellular organization. The progression of hypertrophy is characterized by enlargement of the nuclei, synthesis of new contractile elements and displacement of myofibrils with loss of the normal pattern of the Z-bands. Eventually, the Z-bands are disrupted, the normal arrangement of sarcomeres is disturbed and the contractile elements are lost (myocytolysis). Myocyte loss through different cell death pathways may contribute to progressive remodeling. Important changes also occur in the extracellular matrix with synthesis of collagen (myocardial fibrosis) and impaired crosslinking of myocytes. (1)

With the progression of hypertrophy and remodeling, the excitationcontraction coupling is hampered. Due to the imbalance and impairment of ion channels and exchangers for $\mathrm{Ca}^{2+}$ and other electrolytes as well as abnormalities in contractile and regulatory proteins, the amount of $\mathrm{Ca}^{2+}$ in the myocyte is decreased and levels of diastolic $\mathrm{Ca}^{2+}$ is increased (1). This is manifested in reduced contraction and relaxation, especially with higher heart rates (force-frequency relationship).

In general, elevated wall stress leads to an increased oxygen consumption by the myocardium. Furthermore, when using a simplification of Laplace's law, the larger LV radius - the higher wall stress (1). As mentioned, the wall stress exerted on the LV is a major factor in LV remodeling and hypertrophy (26). Thus, in the dilated ventricle, wall tension is increased raising the oxygen consumption which is already compromised in the failing myocardium $(1,22)$. Also, the increase in myocardial mass is not matched by formation of new blood vessels (neoangiogenesis). With time as the hypertrophied and failing heart is not able to supply sufficient amount of blood, the patient develops decompensated heart failure.

Furthermore, the remodeled heart not only increases in size but the shape also changes from normally elliptical to more spherical (27). LV enddiastolic volume (LVEDV) and wall stress increases and this creates a new mechanical burden for the failing heart (28). LV remodeling results in increased afterload on the heart which stimulates further growth. This generates a vicious cycle of successively increased wall stress, in a downward spiral to adverse cardiac remodeling and heart failure.

All of the aforementioned structural and chemical changes contribute to worsening of cardiac function with progressive hypertrophy, loss of contractile function and myocardial fibrosis during remodeling. Most cases of heart failure are due to inadequate myocardial contractile function, called systolic dysfunction, which is a consequence of e.g. ischemic heart 
disease or cardiomyopathies. LV hypertrophy and myocardial fibrosis can also lead to inability of the heart to adequately relax and fill, called diastolic dysfunction. Often, both systolic and diastolic dysfunction are present in a heart failure patient. Diastolic function can substantially contribute to the pathophysiology of heart failure (1). As a response to the reduction of ventricular compliance, the atrium tries to compensate by increasing in contractile strength and the atrial pressure increases (29).

\subsection{Reverse remodeling}

The progress of heart failure may be viewed as a result of overexpression of biologically active molecules that through various mechanisms change the myocytes phenotype causing deleterious effects on the heart. The target for treatment of heart failure patients is blocking of key neurohormones with angiotensin-converting enzyme inhibitors, angiotensin-receptor antagonists and beta-receptor blockers (30-33). In some patients, LV volume decreases and the shape of the LV return to elliptical following medical treatment (reverse remodeling) (34). Although survival for heart failure patients has been improved, many patients remain symptomatic despite optimized medication.

\subsection{Decompensated heart failure}

Without proper treatment, heart failure patients will in time decompensate and develop symptoms. The failing heart can no longer maintain sufficient cardiac output. This inadequacy is called forward failure and the patient can feel fatigue and tiredness. Forward failure is almost always accompanied by congestion of venous circulation due to increased venous pressure. This is called backward failure and lead to volume retention which is manifested by dyspnoea due to pulmonary congestion and/or oedema due to systemic congestion.

\subsection{Cardiomyopathies}

Contrary to most cardiac diseases that are secondary to other disorders, such as coronary artery disease, hypertension or valvular disease, cardiomyopathies are a group of diseases that affect the cardiac myocardium. They have traditionally been classified into three patterns: 
dilated cardiomyopathy (DCM or DCMP), hypertrophic cardiomyopathy and restrictive cardiomyopathy $(1,22)$. Of these, DCM is the most common and it is primarily characterized by LV dilation and systolic dysfunction. DCM can have several causes including genetic, infectious and toxic exposure. However, in most cases no cause is found, termed idiopathic DCM.

Ischemic cardiomyopathy (ICM or ICMP) is in the vast majority of cases secondary to reduced coronary flow, due to atherosclerotic lesions in the coronary arteries, and it is characterized primarily by LV dysfunction.

Patients with idiopathic DCM are included in paper II-IV and patients with ICM in paper III-IV.

\subsection{Mechanical dyssynchrony}

Deterioration of cardiac function in heart failure is often associated with prolongation of the QRS complex on electrocardiogram (ECG) which reflects disturbed and delayed propagation of electrical signal through the cardiac conductive system. Delayed LV contraction, as in left bundle branch block (LBBB), or RV contraction, as in right bundle branch block (RBBB), result in a mechanical dyssynchrony. Cardiac resynchronization therapy (CRT) is a form of biventricular pacing which aim at restoring synchrony. It is an established therapy for chronic heart failure patients with mechanical dyssynchrony and for those who do not improve on medication. CRT can reduce symptoms and even reverse remodeling in heart failure. However a significant proportion of patients with this treatment do not improve, reasons for which are unknown (35).

Heart failure patients with LBBB are included in paper III and IV.

\subsection{Physiological remodeling}

Moderate exercise is also associated with cardiomyocyte hypertrophy. In contrast to pathological cardiac hypertrophy, exercise-induced hypertrophy is characterized by a physiological heart growth with normal cardiac microstructure (Fig.4) and normal or improved cardiac function (36). In endurance trained athletes with physiologic cardiac remodeling, cardiovascular magnetic resonance imaging shows consistently RV and 
LV cavity enlargement, which maintains the normal shape, in the absence of localized wall thickening (37). Restructuring of the myocardium by exercise is also a balanced increase of myocardial mass between myocyte hypertrophy and formation of blood vessels (38). This kind of exerciseinduced hypertrophy is often referred to as the athlete's heart. This balanced myocardial hypertrophy has been linked to key signaling pathway in the myocytes. Studies on mice have identified exercise-activated cardiac progenitor cells and that cardiac growth is also promoted by formation of new cardiomyocytes (39). 


\section{BLOOD FLOW}

The primary function of the cardiovascular system is to propel blood and maintain blood flow to all parts of the body. Blood flow throughout the cardiovascular system is driven by pressure differences which is generated by the pumping of the heart. Blood is a corpuscular material and acts like a fluid that can be deformed.

\subsection{Flow characteristics}

The flowing blood is subject to friction caused by viscosity between the blood and the boundaries (vessel wall or heart cavity), and also between different layers of streams, called shear stress (40). Wall shear stress is a force acting parallel to the surface, which is proportional to the viscosity of blood and the spatial derivative of the velocity normal to the wall. It is measured in force per unit area. Near the wall the rate of shear stress increases between different layers of stream.

Generally, in smaller vessels, different layers of streams are parallel to each other and the flow is called laminar (Fig.5). It is characterized by smooth streamlines and highly ordered motion with no disturbances.

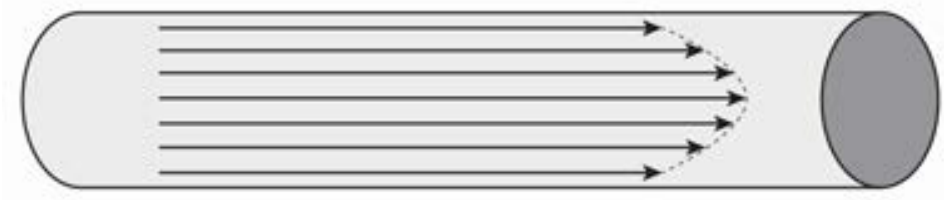

Laminar flow

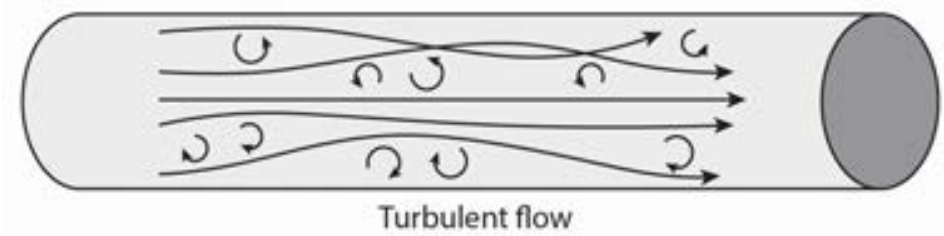

Figure 5: Laminar (top) and turbulent flow (bottom).

In large vessels or chambers of the heart, flow is often unsteady. The transition from steady to unsteady flow, called turbulent flow (Fig.5), is based on Reynolds number which depends on the flow velocity, the vessel 
diameter, and the density and viscosity of the blood $(8,40)$. Turbulent flow is characterized by chaotic and random fluid fluctuations. It is also associated with a high degree of mixing of blood, formation of vortices, and energy losses.

A vortex can be described as a group of fluid particles with a circular/swirling motion around a common axis. Vorticity is a measure of fluid rotation and a vortex can be considered as the local accumulation of vorticity. Vorticity develops as a consequence of velocity difference between the flow and its boundary or in significant shear layers in the fluid. It often occurs due to sharp expansions or narrowings as in presence of a stenosis (41). Vortices are not exclusive to turbulent flow but can also be present in laminar flow. The presence of vortices influences pressure distribution and shear stress and is of vital importance for relating blood motion to pathology (40). Ventricular vortex formation have been ascribed a beneficial role in terms of energy preservation (42).

\subsection{Altered flow}

The interaction between blood flow and the surface of the surrounding vessel or heart cavity has important consequences (43). Lesions and atherosclerotic plaques are often found in locations where blood flow is altered, such as bifurcations or curvatures. Studies of vascular biology have shown association of flow disturbance with vascular fate. In a study on mice (44), the blood flow and shear stress were manipulated in different ways in the carotid arteries and, after a time, the effects of atherosclerosis were compared. The pattern of atherosclerotic plaques were found to be different, despite the fact that the geometry and total flow were identical in the arteries. Changes in shear stress was found to be tightly related to pattern of atherosclerosis.

By only describing the vessel by its geometry and total flow, the important aspects of flow such as shear stress and vortices are missed. This means that the region that traditionally would have been termed normal by anatomical measures, could actually be the region with highest flow abnormality and therefore would be the most vulnerable to disease. Instead of relying exclusively on anatomic description, increasing evidence suggest that flow should be a primary focus by which we explain pathology, guide intervention and form individual therapy based on restoration of flow (43). 


\subsection{Flow-induced forces}

Blood accelerates from areas of high to low pressure. At the start of diastole, the pressure difference between the LA and the LV causes the inflowing blood from the LA to accelerate and enter the LV. In the LV, the myocardium exerts a force to decelerate the blood and at the same time the blood exerts a hemodynamic force with equal magnitude but opposite direction on the LV. This can be explained by Newton's third law (the action-reaction law). After the atrial contraction in late diastole, there is similar pattern of hemodynamic forces. During systole, the contracting myocardium exerts a force to accelerate the blood toward LV outflow tract while the blood exerts a hemodynamic force on the surrounding myocardium.

The blood flow plays an important role in the normal growth and development of the fetal heart. Genes within cells contain the genetic program which dictates the differentiation of the cells that will eventually constitute the heart (cardiogenesis). As blood begins to flow in the developing heart, flow induces a force on the myocardial wall. Cardiac cells can sense the wall shear stress and transmural pressure and respond by changing the gene expressions and organization of the cytoskeletal structure. Hemodynamics is thus an epigenetic factor, which results in change of the shape of the heart (cardiac morphogenesis). The importance of this interplay between the pattern of blood flow and genetic factors has been demonstrated in studies where disturbed flow patterns caused abnormal heart formation in zebrafish embryos (7). Flow dictates the development of the cardiac form and shape.

Early stages of cardiac remodeling can alter the ventricular flow patterns and flow-induced forces induce changes in the heart cells that could lead to progressive pathological remodeling in the failing heart (45). Assessment of the relationship between ventricular morphology and blood flow patterns in healthy and failing hearts may increase the understanding of progressive myocardial remodeling. Findings of abnormal flow may precede detectable morphological changes in the progress of deteriorating cardiac function and could lead to early diagnosis. Different patterns of flow in heart failure patients with otherwise similar echocardiographic indices may explain the different degree of symptoms that these patients present with (46).

Studies of intraventricular flow, using magnetic resonance velocity mapping, have proposed that the curvature of the heart cavities have 
hemodynamic advantages (47). Blood flows through the heart in an asymmetrical and direction-changing fashion that restrict interaction of different streams of flow. Flow instabilities and dissipation of energy are limited and inflowing blood is efficiently redirected towards the next cavity. 


\section{CARDIAC IMAGING \& ASSESSMENT OF CARDIAC FUNCTION}

Noninvasive cardiac imaging is of vital importance in the assessment of several cardiac diseases. Imaging can contribute to key information for the diagnosis of heart failure and has in many cases aided in explaining the etiology of cardiac dysfunction. Efficacy of treatment may also be assessed and guide further treatment.

Cardiac imaging can be performed using different modalities. Echocardiography, cardiovascular magnetic resonance imaging (CMR), nuclear imaging methods and cardiac computed tomography (CT).

Radionuclide imaging is mainly used in cardiac imaging to noninvasively evaluate the myocardial perfusion and viability. The most commonly performed procedure is single photon emission computed tomography (SPECT) in which the injected radiotracer (isotope) is extracted from the blood by viable myocytes. The magnitude of tracer uptake is related to perfusion and provide myocardial perfusion imaging. Cardiac CT is mainly used in the evaluation of coronary arteries but also enables assessment of cardiac volumes and tissue characterization.

\subsection{Echocardiography}

Echocardiography, based on ultrasonography, is a fast and readily accessible noninvasive method to access cardiac structure and function and it is the most widely used clinical tool for cardiovascular flow assessment, especially in emergency cases.

Using Doppler mode, the blood flow velocity component in the direction of the ultrasound beam can be measured and with color Doppler, 2dimensional (2D) visualization of blood flow velocities in one direction can be created $(48,49)$. Doppler echocardiography can thus measure velocity in only one spatial direction over time.

Echocardiographic particle image velocimetry (echo-PIV) is a technique based on 2D echocardiography that can assess flow in two directions (inplane flow components) and measure velocity fields. Acquiring the velocity vector is done by detecting the distance traveled of contrast agent microbubbles over two consecutive time frames and divide it by the time interval. Echocardiography is not able to describe the complex three- 
dimensional and time-varying characteristics of blood flow within the beating heart. Not even reconstruction of single velocity-encoded directions into multidimensional images solves this problem.

\subsection{Cardiovascular magnetic resonance imaging}

CMR is based on magnetic resonance imaging (MRI). It has a unique potential of combining high quality tomographic imaging of the cardiovascular system with qualitative and quantitative evaluation of blood flow within any direction. CMR has the capability of advanced tissue characterization and it provides morphologic and functional information relevant to a broad array of cardiovascular diseases. CMR is not based on ionizing radiation as opposed to cardiac CT. However, CMR has limitations when patients have certain metallic implants creating image artifacts and safety issues.

\subsection{Assessment of cardiac function}

Assessment of cardiac function is a complex process where many different measures of systolic and diastolic ventricular function are used. One of the most clinically used measure of systolic ventricular function is the ejection fraction (EF). EF is defined as the fraction of the EDV that is ejected during systole, i.e. EF is the stroke volume divided by EDV. Other measures of ventricular systolic function are atrioventricular plane displacement, myocardial systolic velocities and myocardial systolic deformation.

The assessment of LV diastolic function is often performed by echocardiography using parameters such as transmitral and pulmonary venous inflow patterns (25). E-wave (E) is the transmitral inflow during early diastolic filling and A-wave (A) is the inflow during late diastolic filling. Traditional classification of diastolic dysfunction has been based on patterns of these waves (Fig.6). At the early stage of diastolic dysfunction, ventricular relaxation is impaired and the E-wave becomes smaller. Simultaneously, A-wave increases as the strength of the atrium increases. This is the typical appearance in the state of impaired relaxation or mild dysfunction (grade I diastolic dysfunction). As diastolic function continues to deteriorate, the E-wave increases due to an increase in LA pressure. The A-wave decreases because of increased ventricular pressure and beginning of atrial dysfunction. The E/A-wave pattern and ratio revert 
to relatively normal, called pseudonormal (grade II diastolic dysfunction), and it can be hard to distinguish from normal conditions. Further deteriorating diastolic function leads to a marked increase in the E-wave with a steep descending slope. This pattern is called restrictive and indicate severe diastolic dysfunction including significantly elevated LV filling pressures (grade III diastolic dysfunction).

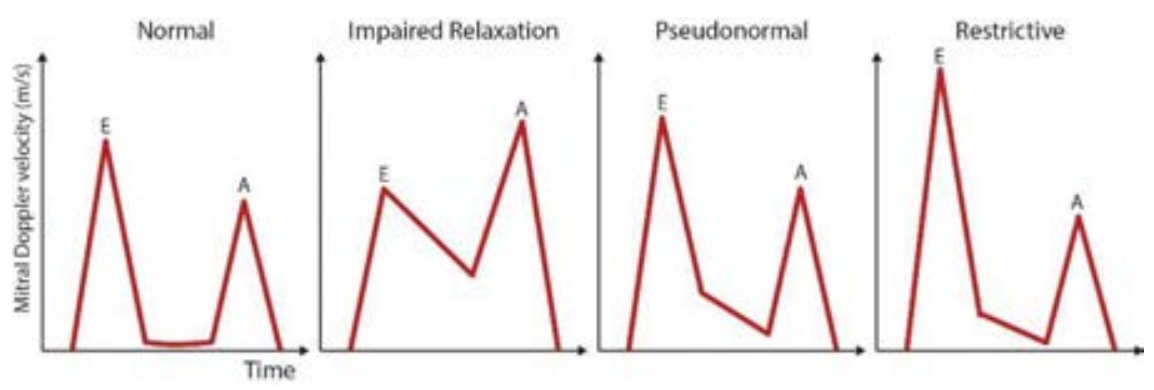

Figure 6: Transmitral Doppler LV inflow velocity in normal hearts and in failing hearts with different degrees of diastolic dysfunction.

Doppler echocardiography measurements are often based on assumptions about flow profiles and vessel morphology to give an estimation of the total flow. However, important but complex flow patterns can be omitted, which may result in incorrect flow quantification, especially in areas where lumen geometry is complex such as in the ventricles. Furthermore, Doppler imaging is limited by variable velocity assessment due to beam alignment, it requires an acoustic window and is dependent on operator expertise. 
MRI is based on the phenomenon of nuclear magnetic resonance, in which certain atomic nuclei, with an odd number of protons and/or neutrons, absorbs and emits a radio frequency signal when placed in a magnetic field (50). One such atomic nucleus is the proton of the hydrogen atom $\left({ }^{1} \mathrm{H}\right)$ which is abundant in the human body in the form of water and fat molecules. MRI techniques rely on protons of ${ }^{1} \mathrm{H}$ to generate a signal that will yield an image.

\subsection{Spin}

As all elementary particles, the hydrogen proton possesses a magnetic momentum, called spin $(\mu)$, due to its rotation along its axis (Fig.7). Placed in a magnetic field $\left(\mathrm{B}_{0}\right)$, spins of many protons will rotate either parallel or antiparallel about the magnetic field direction of $\mathrm{B}_{0}$ ( $\mathrm{z}$ direction of a Cartesian 3D coordinate system) like a gyroscope with a fixed precession frequency. This frequency is called Larmor frequency and it is proportional to the strength of the magnetic field. In a strong magnetic field, as in the MRI scanner, there is a small net excess of spins parallel to the magnetic field because this orientation requires less energy. This small excess of parallel-oriented spins form a net magnetization $\mathrm{M}_{0}$ pointing in the $+\mathrm{z}$ direction (Fig.7 and Fig.8A).
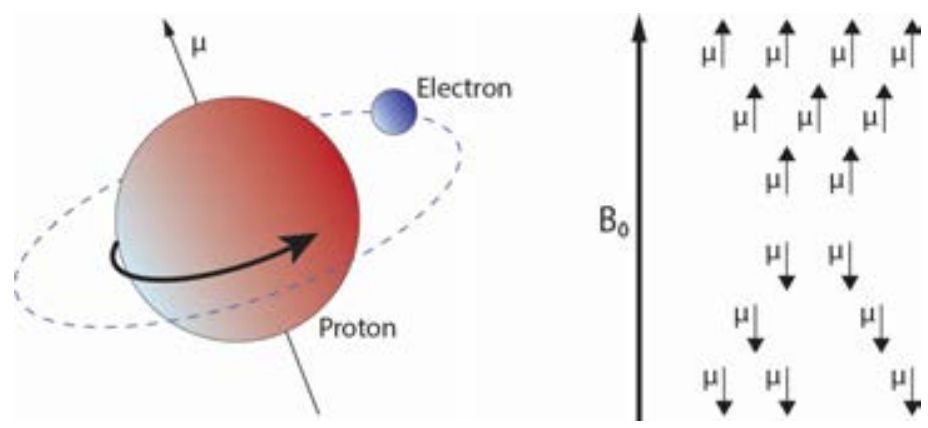

Figure 7: The proton and its spin, $\mu$ (left). Excess of spins parallel to external magnetic field $\mathrm{B}_{0}$ (right). 


\subsection{Radio frequency pulse}

During the measurement process, the patient is exposed to a brief radio frequency (RF) pulse which matches the Larmor frequency of the spins. The RF will be absorbed by the spins, called resonance, and the net magnetization $\mathrm{M}_{0}$ will be tilted away from the $+\mathrm{z}$ direction (Fig. $8 \mathrm{~B}$ ). The amount of resulting rotation of $\mathrm{M}_{0}$ relative to $\mathrm{B}_{0}$ is known as the pulse flip angle $(\alpha)$. During this change of direction, the magnetization $\mathrm{M}_{0}$ can be decomposed into two components: one component aligned with the $+\mathrm{z}$ direction, called longitudinal magnetization $\left(\mathrm{M}_{\mathrm{z}}\right)$ and the other component lying on a plane perpendicular to the $+\mathrm{z}$ direction (xy plane of a Cartesian $3 \mathrm{D}$ coordinate system), called transverse magnetization $\left(\mathrm{M}_{\mathrm{xy}}\right)$. As the spins start to tilt, $\mathrm{M}_{0}$ rotate away from $+\mathrm{z}$ direction and will eventually align on xy plane (Fig. $8 \mathrm{C}$ ). This is called a $90^{\circ} \mathrm{RF}$ pulse.
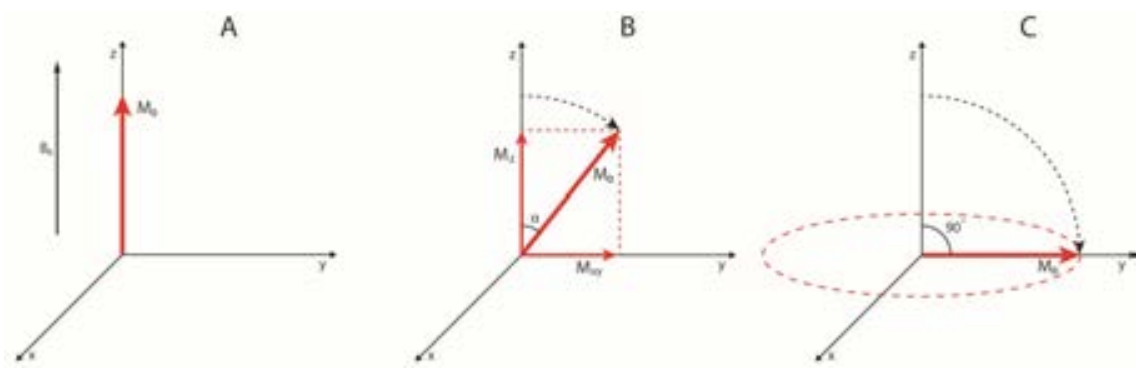

Figure 8: Net magnetization $\mathrm{M}_{0}$ in the $\mathrm{z}$ direction of a Cartesian $3 \mathrm{D}$ coordinate system (A). $\mathrm{M}_{0}$ decomposed into $\mathrm{M}_{\mathrm{z}}$ and $\mathrm{M}_{\mathrm{xy}}$ (B). A $90^{\circ} \mathrm{RF}$ pulse flips the net magnetization $\mathrm{M}_{0}$ from $+\mathrm{z}$ direction onto xy plane (C).

\subsection{Relaxation time $T 1 \& \mathrm{~T} 2$}

As soon as the RF pulse is removed, spins will start to return to the original orientation and emit a signal, called free-induction decay (FID) that can be detected and recorded. The spins decay with time as more and more of the protons give up their absorbed energy through a process called relaxation. The relaxation is a result of two processes: (I) energy between spins and its surrounding molecules (spin-lattice interaction) will cause the magnitude of $\mathrm{M}_{\mathrm{z}}$ to increase towards $\mathrm{M}_{0}$, called longitudinal relaxation (Fig.9); (II) energy exchange between spins (spin-spin interaction) will cause dephasing along the xy plane and $\mathrm{M}_{\mathrm{xy}}$ decreases to zero, called transverse relaxation (Fig.10). 

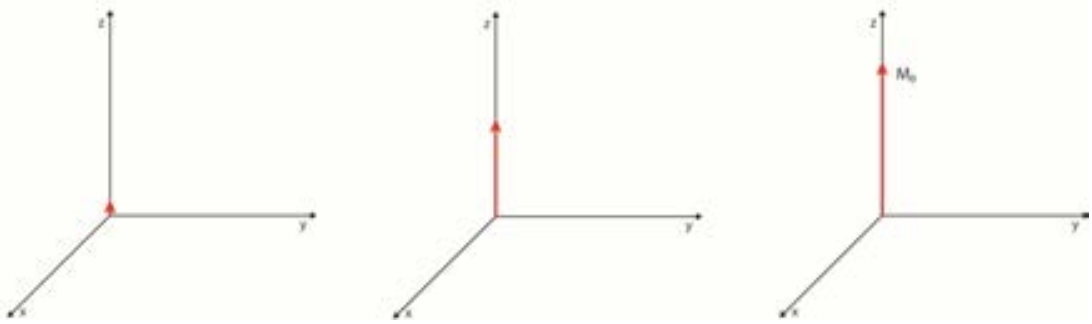

Figure 9: Net magnetization $\mathrm{M}_{0}$ during $\mathrm{T} 1$ relaxation.
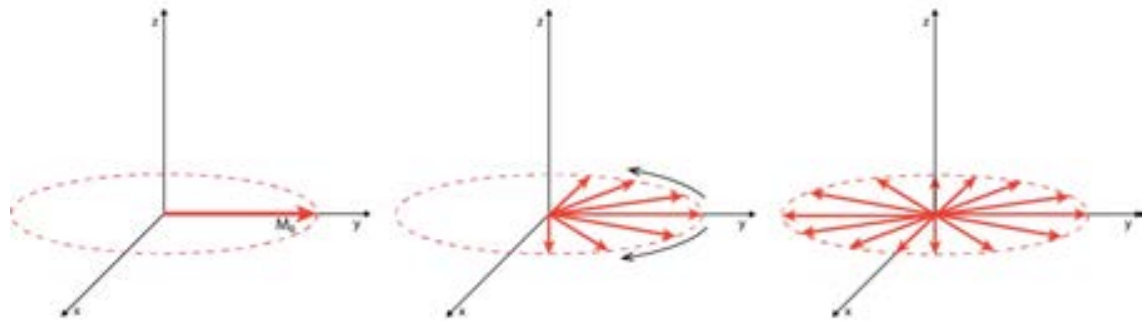

Figure 10: Net magnetization on the xy plane during T2 relaxation.

$\mathrm{T} 1$ relaxation is the time it takes for $\mathrm{M}_{\mathrm{z}}$ to increase to $63 \%$ of $\mathrm{B}_{0}$, from end of the RF pulse. $\mathrm{T} 2$ relaxation is the time for $\mathrm{M}_{\mathrm{xy}}$ to decrease $37 \%$. In reality, there are small differences in the static magnetic field at different spatial locations, called inhomogeneities, which will cause the transverse $\mathrm{T} 2$ relaxation to be faster. $\mathrm{T} 2 *$ relaxation takes into account the inhomogeneity of the static magnetic field $\mathrm{B}_{0}$.

MRI takes advantage of the fact that hydrogen atoms in different molecular environment have different magnetic properties. Water has two hydrogen atoms bonded to one oxygen atom, whereas fat is heterogeneous in nature, with many hydrogen atoms bonded to a long-chain carbon framework. Because of this, a water proton has a different local magnetic field than a fat proton. The values of $\mathrm{T} 1$ and $\mathrm{T} 2$ relaxation depend on the unique inherent properties of the tissue and these differences provide the source of contrast in the MRI image. 


\subsection{Pulse sequences}

A trail of RF pulses is called a pulse sequence. Different pulse sequences will produce distinct images with different contrast depending on imaging parameters and tissue $\mathrm{T} 1$ and $\mathrm{T} 2$ relaxation values.

Commonly used sequence in CMR is spin-echo (SE) sequence. First a $90^{\circ}$ RF pulse is applied which brings $\mathrm{M}_{0}$ from $+\mathrm{z}$ direction to the $x y$ plane. After a period of time (TE/2) a $180^{\circ} \mathrm{RF}$ pulse is applied in order to refocus the decaying transverse magnetization and after the same amount of time (TE/2) a signal is produced called spin echo. The time from the $90^{\circ} \mathrm{RF}$ pulse to the signal is called echo time (TE). The procedure is repeated and the time between two $90^{\circ} \mathrm{RF}$ pulses is called repetition time (TR). By using a $180^{\circ} \mathrm{RF}$ pulse in spin-echo sequence, the effect of field inhomogeneity will be eliminated as it will cancel itself out after refocusing. Adjusting the lengths of TE and TR will weight the image contrast toward T1 or T2 relaxation values. A short TR and TE produce a T1 weighted image, and conversely, a long TR and TE produce a T2 weighted image.

Another important sequence is gradient-echo (GRE). $\mathrm{M}_{0}$ is tipped away from $+\mathrm{z}$ direction only by a small angle by the use of small flip angles RF instead of $90^{\circ} \mathrm{RF}$ pulses. The reason is to shorten the time for longitudinal relaxation, which may be important in order to produce many signals during a short time interval. The decaying transverse magnetization is refocused by a bipolar magnetic gradient instead of a $180^{\circ} \mathrm{RF}$ pulse. This produces a gradient echo. Compared to spin-echo, scan time is significantly reduced by avoiding the use of $90^{\circ}$ and $180^{\circ} \mathrm{RF}$ pulses. TR in the GRE sequence can be as short as few milliseconds. Signal intensity and image contrast is determined by the choice of pulse sequence and the flip angle used.

\subsection{Encoding of MRI signal \& k-space}

The MRI signal needs to be spatially encoded in order to determine wherefrom the signal is originating. This is achieved in three ways. First, rather than having a spatially uniform magnetic field $\mathrm{B}_{0}$, a magnetic field gradient is introduced along the z-axis (Fig.11A) and by tailoring the RF pulse, a plane along the $\mathrm{z}$ axis can be selected. Only spins in that slice will be on-resonance with the RF pulse and flip. Secondly, frequency-encoding is induced by a magnetic field gradient along the x-axis (Fig.11B). As a 
result, spins along the $\mathrm{x}$-axis will have known different frequencies. Thirdly and lastly, the y-axis is phase-encoded by applying different phases continuously along the y-axis (Fig.11C).
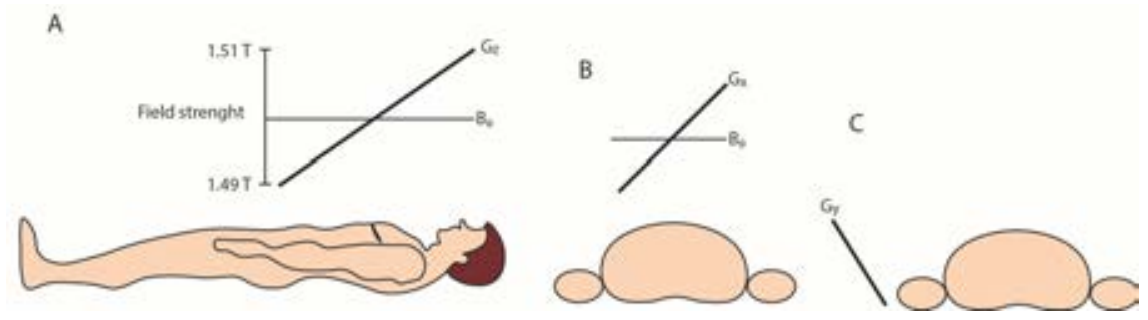

Figure 11: Slice selection (A), phase encoding (B) and frequency encoding (C).

The frequency- and phase-encoded MRI signal in every plane is recorded sequentially in k-space, which is a two-dimensional data matrix with the $\mathrm{x}$-axis representing the frequency encoding and $\mathrm{y}$-axis representing the phase encoding. The center of $k$-space holds the bulk of image contrast and the periphery holds the edges and details of the image. By using the Fourier transformation, the k-space can be converted into an anatomical image.

\subsection{T versus 1.5T MR}

The strength of a magnetic field in a MR scanner is typically measured in units of tesla $(\mathrm{T})$. The main advantage of the stronger magnetic field in a 3T MR scanner is higher signal-to-noise ratio (SNR) which is approximately twice that of $1.5 \mathrm{~T}$ scanner. This can be used to improve image quality or reduce scan time. Increased SNR leads to further improvements such as the ability to increase resolution in a plane or decrease slice thickness, improving image clarity. A higher SNR can also be used to improve temporal resolution and decrease acquisition time. Artifacts resulting from breathing or any type of motion including flowing blood are more prominent on 3.0T versus 1.5T MR scanners (50). 


\section{CARDIOVASCULAR MAGNETIC RESONANCE IMAGING (CMR)}

In recent years, CMR has become the gold standard in the noninvasive measurement of both left and right heart volumes and for flow volume quantification (51). Clinical CMR techniques enable assessment of cardiac morphology, characterization of myocardial tissue including myocardial oedema and fibrosis, as well as assessment of myocardial strain, myocardial perfusion and intracardiac blood flow.

\subsection{Cardiac gating}

Contrary to a static tissue at rest, the heart muscle is continuously moving. Standard MR acquisitions are too slow to capture the dynamic cardiac motion in real time with sufficient spatial resolution. Scan acquisition has to be synchronized with the different cardiac phases, otherwise the myocardial contraction and relaxation and the pulsatile blood flow will blur the CMR image. Cardiac gating is therefore an essential component of CMR and it is done by the aid of ECG registering. In this way, segments of k-space are obtained at the same time-point within the cardiac cycle over many sequential heartbeats.

CMR acquisition can be gated prospectively or retrospectively. In the former case, the R-peak in the QRS-complex of the ECG triggers the data acquisition at the start of the cardiac cycle and in the latter case, data is continuously collected over several cardiac cycles and in post-processing this data can be reconstructed. In prospective gating the end of each cardiac cycle is lost due to the fact that acquisition is interrupted to await the next cardiac phase. This affects, for instance, the assessment of cardiac volumes and function. Also there is a trigger delay at the beginning of the cardiac cycle between the registration of the QRS-complex and the initiation of the data collection.

Retrospective gating on the other hand tends to lead to more temporal blurring because each cardiac cycle is interpolated onto a set number of equally spaced phases in data reconstruction despite the fact that the actual $\mathrm{R}-\mathrm{R}$ interval may vary. However, it is more robust than prospective gating, as the signal intensity throughout the cardiac cycle is uniform due to no interruption of excitation pulses. 


\subsection{Respiratory gating}

Breathing motion induces blurring in CMR images. This problem can be tackled by image acquisition during breath-hold which is limited by the capacity of the patient. However even in a patient with excellent breathinghold capacities, the position of the heart at each breath-hold may vary. Breath-holds at end-tidal volumes is widely used to reduce variability.

Another method is registering the breathing pattern by respiratory gating using bellows that measure strain around the patient's belly or respiratory navigator that monitor the diaphragm motion. Acquisition is then only done during consistent part of the respiratory phase or in the case of continuous data acquisition, the data acquired outside a predefined respiratory window is rejected in the post-processing. However, respiratory gating leads to longer acquisition time and this may induce the risk of patient movement or progressive drifting of diaphragm position.

\subsection{Acquisition time}

There is a tradeoff between spatial and temporal resolution in order to keep the total acquisition time as short as possible. Higher spatial resolution images will require more phase-encoding steps and subsequently a larger number of views (or lines of k-space) per cardiac phase (segment) if the scan time should be kept the same. This will lead to a decrease in temporal resolution as more time is taken to acquire each segment. Regarding temporal resolution, only a limited number of segments can be acquired with each cycle as the R-R interval is finite, and thus the number of cardiac phases that can be imaged is limited.

\subsection{Balanced steady-state free-precession}

Images of the heart in motion for visualization and quantification of cardiac anatomy and function uses a cine MRI technique that takes images of the heart in motion throughout the cardiac cycle. Balanced Steady-State FreePrecession (bSSFP) is a form of rapid GRE, with retrospective ECGgating, in which the residual transverse magnetization is used in the subsequent excitation/refocusing cycle (50). The residual transverse magnetization does not decay completely by the next excitation because the TR is minimized. At the next excitation, the recovered longitudinal 
magnetization will be added to the residual transverse magnetization. After several cycles, a steady state is achieved between the longitudinal and residual magnetizations. The signal recorded will consist of two components: FID of the newly excited longitudinal magnetization (T1 - and $\mathrm{T} 2 *$-weighted), and refocused residual transverse magnetization (T2weighted). This produces a unique mixture of $\mathrm{T} 1$ and $\mathrm{T} 2$ and a high contrast between myocardium and the bright blood. The signal-to-noise ratio (SNR) is high as well as spatial resolution, and acquisition times are rapid. During bSSFP, standardized long-axis (LAx) cine images including two-chamber (2ch), three-chamber (3ch) and four-chamber (4ch) views are acquired as well as a stack of short-axis (SAx) views (Fig.12).

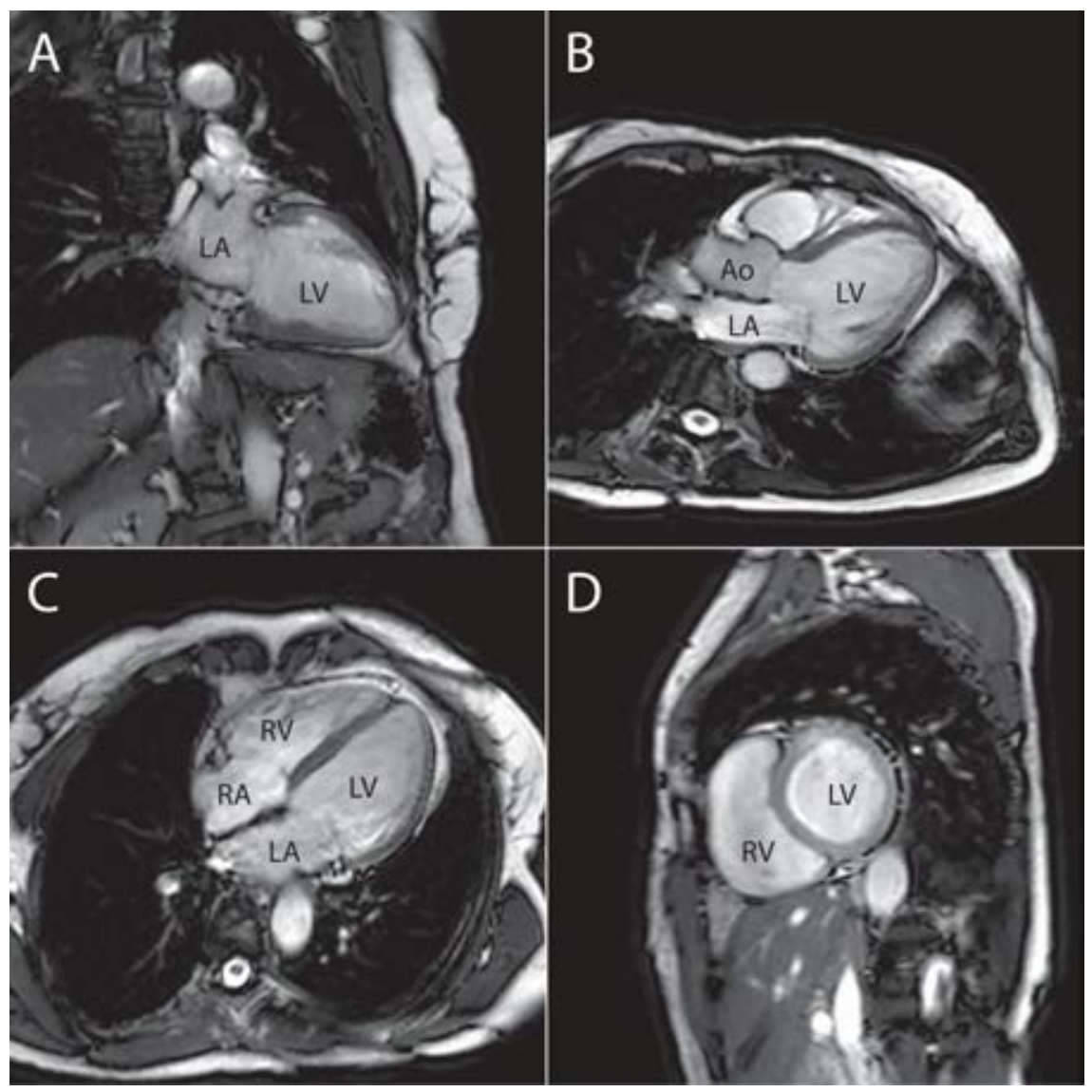

Figure 12: Two-chamber (A), three-chamber (B), four-chamber (C) and midventricular short-axis (D) cine images. Ao, aorta; LA, left atrium; LV, left ventricle; $R A$, right atrium; $R V$, right ventricle. 


\section{4D FLOW CMR}

Velocity-encoding in a single direction based on CMR (2D cine phasecontrast CMR) is widely used at centers for flow volume quantification and estimation of different forms of pathological flows, for example shunt flows or regurgitant flows $(52,53)$. In this thesis, 4D Flow CMR was used to assess aspects of the intraventricular flow in both healthy subjects and patients.

\subsection{Phase-contrast MR}

Phase-contrast MR methods are based on the fact that the transverse magnetization of spins that move in the presence of a magnetic field gradient obtain a different phase than static spins (Fig.13). The flowing blood causes a phase shift. By adding bipolar velocity-encoding gradient with equal magnitude and opposite directions to the cardiac-gated GRE sequence, the velocity of the blood flow can be measured (50). This is accomplished by calculation of the phase difference from two acquisitions with different velocity-dependent signal but otherwise identical sequence parameters $(54,55)$. The net phase shift in each voxel of the image is proportional to the flow velocity of spins along the velocity-encoding gradient. Static spin in stationary tissue have an intermediate signal intensity (grey) on a phase-contrast image, while blood have higher or lower signal intensity (white and black respectively), depending on the direction of flow in the velocity-encoding gradient.

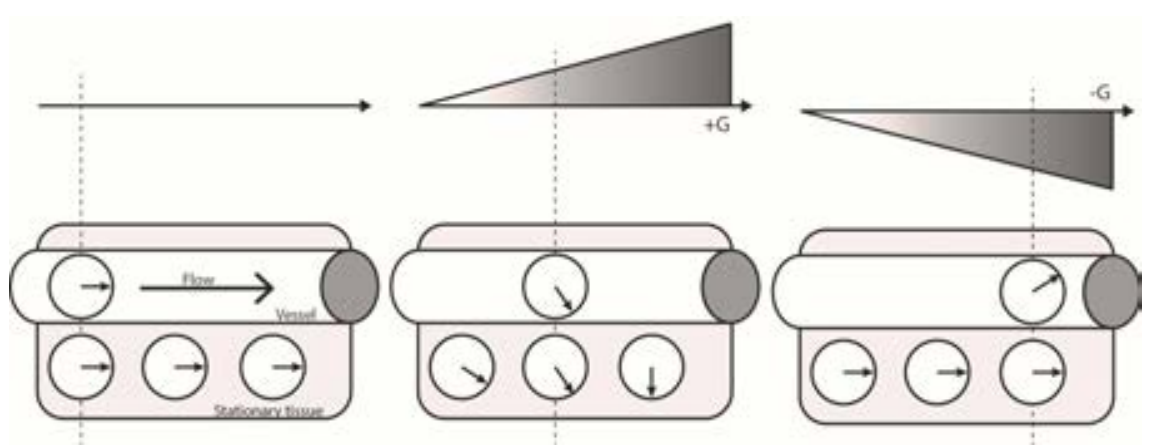

Figure 13: The principle of phase-contrast imaging. After gradient $+\mathrm{G}$ and $-\mathrm{G}$ is played out, spins of moving blood have accumulated a phase shift. 
The magnitude of the velocity encoding gradient has to be adjusted to cover the estimated maximal velocity of the scanned image, otherwise aliasing artifacts will occur. This is called velocity encoding (VENC) and will affect the SNR $(50,54)$. If the VENC is set too high, the different velocities will have poor contrast. There is a trade-off between the minimum detectable velocity due to noise and the maximum velocity that can be detected without aliasing. For optimal noise performance, the VENC should therefore always be as small as possible.

\subsection{D Flow CMR acquisition}

Although CMR can provide excellent spatial resolution images, there are limitations. In reality, the scan time must also be taken under consideration, as higher spatial and temporal resolution requires longer scan times which, in turn, may be impossible for patients with induced risk of motion during acquisition. Apart from these factors, the scan time or acquisition time also depends on other parameters such as magnetic field strength, volumetric coverage, the breathing pattern and compensation efficiency, the heart rate and the applied sequence $(54,56,57)$. Common MRI parameters for cardiac flow measurement is a spatial resolution of $3 \mathrm{~mm}^{3}$ and a temporal resolution of $50 \mathrm{~ms}$, which requires a scan time of approximately 12-15 minutes on a 3 T MRI (58).

4D Flow CMR does not require intravenous administration of any contrast agent, however in the CMR research field, 4D Flow CMR sequences are often run together with other sequences, such as gadolinium late enhancement, that require a contrast agent. The contrast agent is used to improve the image SNR and contrast-to-noise ratio (CNR) in the cine imaging and 4D Flow acquisition.

\subsection{D Flow CMR data analysis}

From phase-contrast imaging, flow-encoding in all three spatial directions can be resolved relative to all three dimensions of space and to the dimension of time $(3 \mathrm{D}+$ time $=4 \mathrm{D})$. From this data, visualization and quantification of the blood flow patterns over an averaged cardiac cycle can be assessed (59). 
To assess the large amount of time-resolved, three-dimensional and threedirectional CMR flow data, it has to be displayed on a two-dimensional screen in order to be visualized. Commonly used techniques for displaying this flow data are streamline and pathline visualization. A streamline is a curve that is everywhere tangent to the instantaneous local velocity vector (Fig.14). Streamlines represent the instantaneous direction of flow through the entire flow field (distance of a vessel or heart cavity) at a specific time point. Regions of recirculating flow and stream layer separation due to turbulent flow can be identified by streamline patterns. A pathline on the other hand is the actual path travelled by an individual particle over a time period (Fig.14). Thus pathlines represent the path travelled by a blood volume and it is the easiest flow pattern to understand. If the velocity vector-field does not change as in steady flow, the streamlines and pathlines would be the same.

A

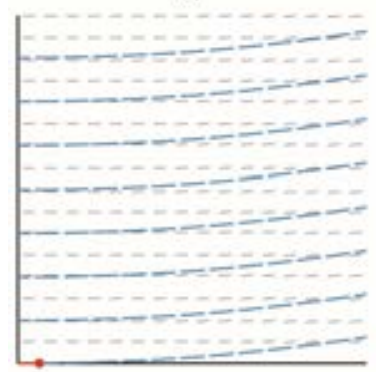

B

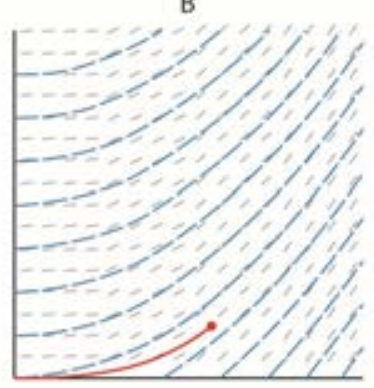

C

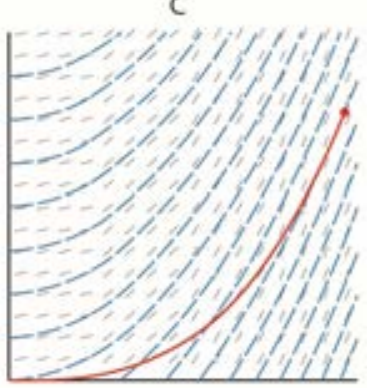

Figure 14: Velocity vectors (grey) of the velocity field, streamlines (blue) and pathline (red) in three subsequent time points A-C.

A validated flow analysis method using pathlines (60-64) was used in paper I to describe the spatial distribution and dynamics of flow in normal RVs and in paper III in dysfunctional LVs with and without mechanical dyssynchrony.

From a segmentation of the end-diastolic volume (EDV), a pathline is emitted from the center of each voxel at ED and traced forwards and backwards to the time of ES, thus encompassing one cardiac cycle. Each pathline is considered to represent a volume of blood corresponding to the voxel size. At end-systole, the segmentation of end-systolic volume (ESV) is used to determine the origin (backwards traces) and destination (forwards traces) of each pathline. The blood volume is divided into four 
functional flow components (Fig.15): Direct Flow: blood that enters the ventricle during diastole and leaves the ventricle during systole in the analyzed heart beat; Retained Inflow: blood that enters the ventricle during diastole but does not leave during systole in the analyzed heart beat; Delayed Ejection Flow: blood that starts and resides inside the ventricle during diastole and leaves during systole in the analyzed heart beat; and Residual Volume: blood that resides within the ventricle for at least two cardiac cycles.
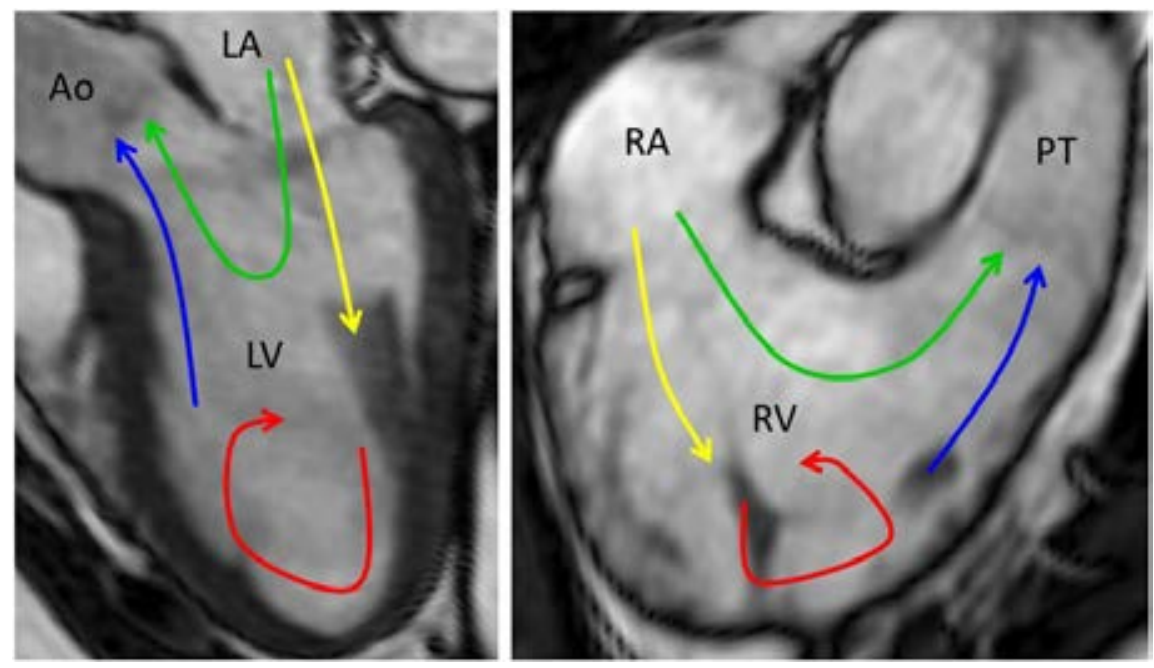

Figure 15: Flow components illustrated in the LV (left) and RV (right): Direct Flow (green), Retained Inflow (yellow), Delayed Ejection Flow (blue) and Residual Volume (red). Ao, aorta; PT, pulmonary trunk.

The inflow components Direct Flow and Retained Inflow can be further divided into early and late inflowing portions according to the two diastolic phases (early and late inflowing Direct Flow, and early and late inflowing Retained Inflow). This division is used in paper III.

By separating the blood flow into flow components, analyses can be performed to measure their volume and quantify the proportion of each component. From the volume occupied by each trace, its velocity and the density of the blood, kinetic energy (KE) can be calculated for each flow component over the cardiac cycle. The KE at the time of ED was used in paper I and III for comparison and considered as the pre-systolic energetic value. 
Turbulent blood flow causes energy loss in the cardiovascular system. TKE is based on the standard deviation of the phase-contrast signal magnitude inside a voxel and is thus an estimated measure of mean velocity variation or velocity fluctuation (65). Calculation of TKE can be used to estimate turbulence-related loss of energy and was used in paper II.

By integrating the pressure gradients over the LV volume at each time frame from 4D Flow CMR data, the magnitude and the direction of the total hemodynamic force between the LV blood and the surrounding myocardial walls was calculated over the cardiac cycle in paper IV. The maximum hemodynamic forces acting in the short axis (SAx) and long axis (LAx) directions were used to calculate the $\mathrm{SAx} / \mathrm{LAx}$-ratio at early and late diastolic filling. The SAx/LAx-ratio reflects the deviation of the LV hemodynamic forces from the main flow direction. The predominant flow direction in the normal LV is along the apex-to-base axis.

\subsection{Artifacts}

There are several artifacts, such as concomitant gradient fields, eddy currents and phase wraps, when using 4D Flow CMR that may lead to errors and they have to be compensated for. Often this kind of correction is part of the reconstruction of the phase-contrast MR image (58). 


\section{4D FLOW CMR ANALYSES \& PRESENT STUDIES}

As previously described the general direction of intracardiac blood flow is orderly and timely determined by the different cardiac phases of atrial and ventricular contraction and relaxation and by the opening and closure of valves depending on pressure gradients. However, there are many different routes by which the blood flow can achieve this and it involves flow directions in all spatial dimensions. This multi-dimensional, multidirectional, pulsatile flow has previously been too complex to access in its entirety. Computational fluid dynamics (CFD) has provided some insights into the physiology of blood flow and the pathophysiology (58).

4D Flow CMR offers positioning of planes or masked volumes at any position for measuring or analyzing flow as well as combining flow visualization with bSSFP cine images for providing a comprehensive image of cardiovascular physiology and also anatomy.

4D Flow CMR has previously been applied for analysis of blood flow in both healthy subjects and patients regarding the ventricles $(47,60,61,63$, $66,67)$ and the atria $(68,69)$ of the heart, heart valves and valve prostheses (70-72), aorta (73-77), pulmonary vessels (78), and other arteries and veins in the body $(79,80)$. Quantification of flow can be performed from measurements of basic blood flow volumes and velocities to advanced estimations of derived hemodynamic markers such as turbulent kinetic energy (TKE) (81-83), pressure gradients (84-86), wall shear stress (87, 88 ) and hemodynamic force (89). Relative pressure field are computed from the velocity field and can estimate pressure differences (84). 


\subsection{Flow analysis in normal right ventricle (paper I)}

Studies on normal LVs have showed that the volume of blood entering and leaving the LV within one heartbeat, i.e. Direct Flow, constitutes approximately one-third of the LVEDV $(37 \pm 5 \%)$ and that it is significantly larger than the three other components (Fig.16) $(62,64)$. At the time of end-diastole, Direct Flow possessed a significantly larger amount of KE $(0.55 \pm 0.16 \mathrm{~mJ})$ than the other components. The findings showed that Direct Flow was proportionally the largest of the flow components, it possessed the highest amount of pre-systolic KE and had the shortest distance and the smallest angle to the left ventricular outflow tract (LVOT) at the time of ED, just before onset of systole. The late inflowing Direct Flow was located closest to LVOT. The KE measured for Direct Flow at ED was preserved following late diastolic filling and atrial contraction.

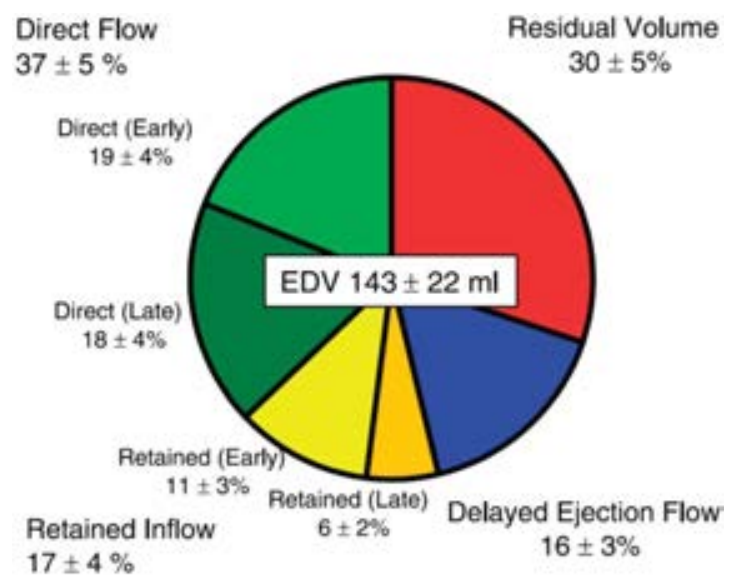

Figure 16: Volume of the LV flow components as a \% of EDV (mean \pm standard deviation). The two inflow components (Direct Flow and Retained Inflow) were also divided into which diastolic phase they entered (early and late inflow). (64)

In paper I, Direct Flow in normal RVs shared some similar characteristics to that of the LV's. It was predominantly located in the basal and midventricular regions. It also had a larger volume $(44 \pm 6 \%)$ and possessed a larger pre-systolic KE $(0.41 \pm 0.34 \mathrm{~mJ})$ than the other flow components. However, compared to LV, RV Direct Flow comprised a larger portion of the EDV and did not extend as much into the apical region of the ventricle (Fig.17). Further, in RV, the Direct Flow followed a smoother curving route than was observed for LV Direct Flow, which was more acute. 


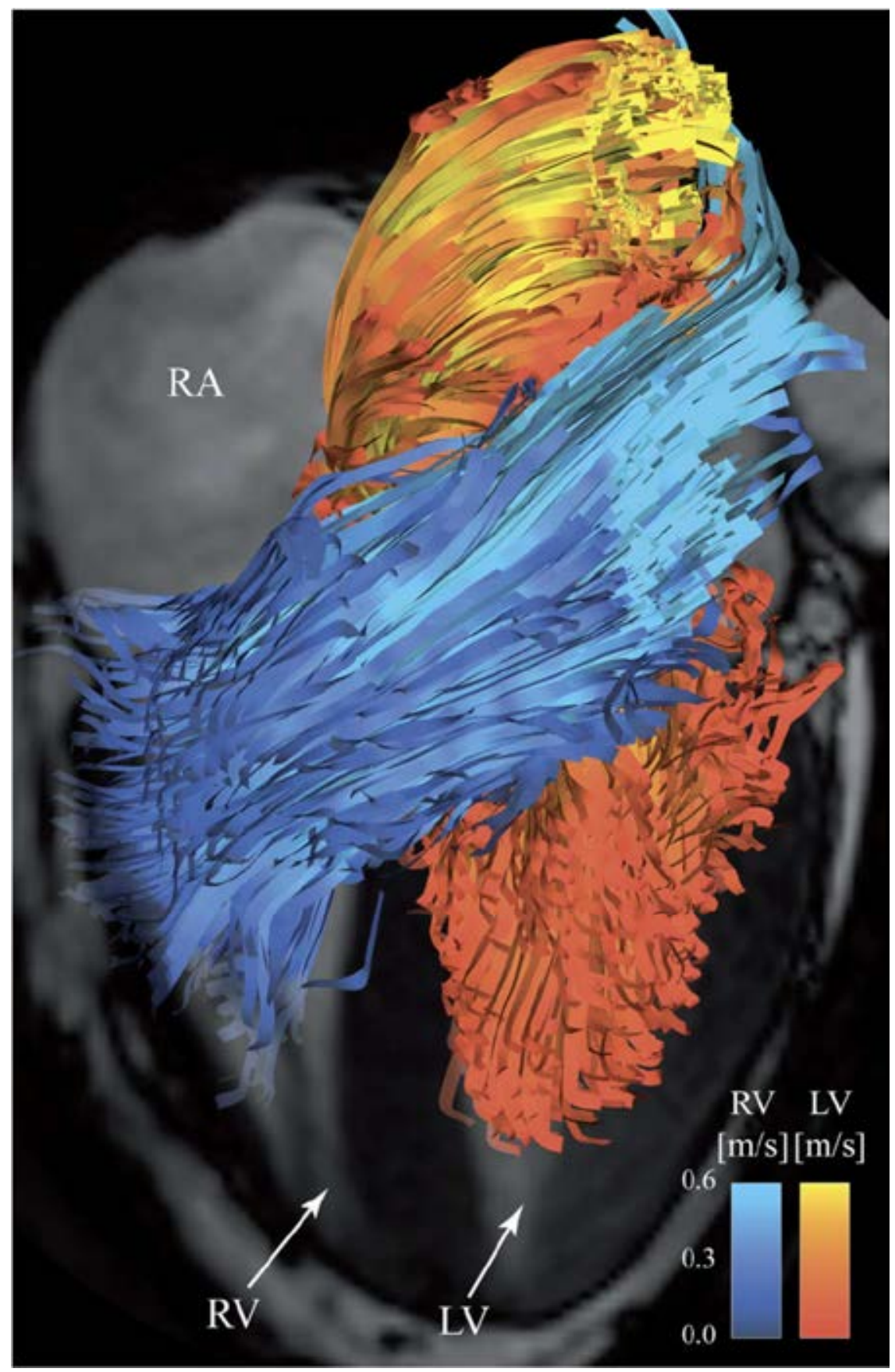

Figure 17: Pathline visualization of Direct Flow in RV (blue) and LV (red). $\mathrm{LV}$, left ventricle; RA, right atrium; RV, right ventricle. 


\subsection{TKE in normal \& myopathic left ventricles (paper II)}

4D Flow CMR was used in paper II to compare TKE values, timing, and distribution within the normal LVs to a spectrum of patients with DCM. Low values of TKE were observed in normal LVs and was predominantly situated in the basal third of the LV, close to the LVOT. In contrast, TKE in DCM patients was observed deeper in the central part of the LV cavity. TKE values in healthy subjects and DCM patients increased during early diastole and were highest just after early filling peak velocity. As the LV continued to fill during late diastole, TKE values in normal LVs diminished (Fig. 18). In LVs of failing hearts, TKE instead increased as the LV size increased during late diastole and exceeded values in normal LVs. In the majority of patients, a peak TKE was observed in late diastole.

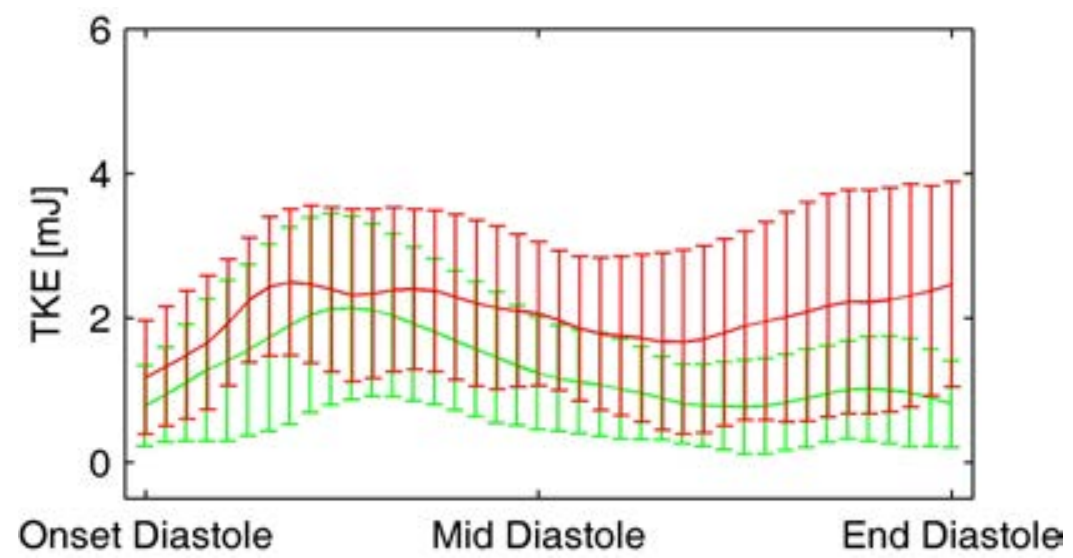

Figure 18: Left ventricular TKE (mean \pm standard deviation) during diastole in DCM patients (red) and in healthy subjects (green).

10.3 Flow analysis in failing hearts with \& without LBBB (paper III)

The organized multidimensional blood flow pattern within the normal heart is altered in heart failure patients $(46,62,90)$. In myopathic LVs, reduced volume and pre-systolic KE of the Direct Flow has been demonstrated compared to normal LVs $(62,91)$. The inflowing Direct Flow proceeds deeper into the LV chamber and interacts more with blood already residing in the LV. The KE loss reflects deceleration, and a larger 
portion of the inflowing blood does not achieve ejection and has to await ejection and reacceleration in the following heartbeat.

Heart failure is often associated with LBBB but how this electromechanical delay influences left ventricular (LV) diastolic function remains unclear. In paper III, using the above mentioned 4D Flow analysis method, the impact of mechanical dyssynchrony on diastolic function was investigated by comparing flow patterns and energetics in heart failure patients with LBBB to matched patients without LBBB.

The volume of the Direct Flow was not different between the groups. However, the pre-systolic KE of Direct Flow was reduced in LBBB patients compared to matched patients with normal conduction (Fig.19). This reduction was attributed to Direct Flow entering during early diastolic filling, whereas no intergroup difference was observed during late filling. This reflects a reduced KE preservation of early inflowing Direct Flow in heart failure patients with LBBB.

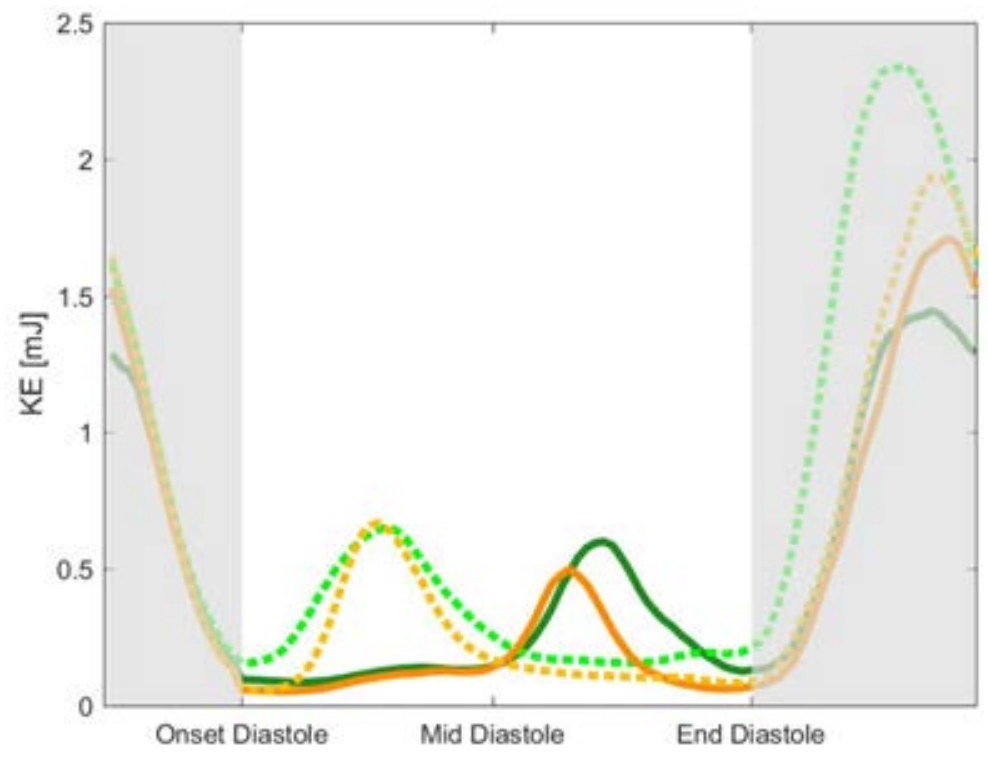

Figure 19: KE (mean) over diastole (white area) and systole (grey areas) for early inflowing Direct Flow (dotted lines) and late inflowing Direct Flow (solid lines) in LBBB patients (orange) and matched patients (green).

Note the larger KE at ED for the early Direct Flow in matched patients compared to that of the LBBB patients. 
10.4 Hemodynamic force in failing hearts with \& without LBBB (paper IV)

Previously hemodynamic force was compared using SAx/LAx-ratio between healthy and failing LVs. In normal subjects, LV hemodynamic force is mainly directed along the base-to-apex axis throughout diastole. In failing LVs, the force is to a greater extent orthogonally oriented to the main flow direction compared to normal LVs (Fig.20).

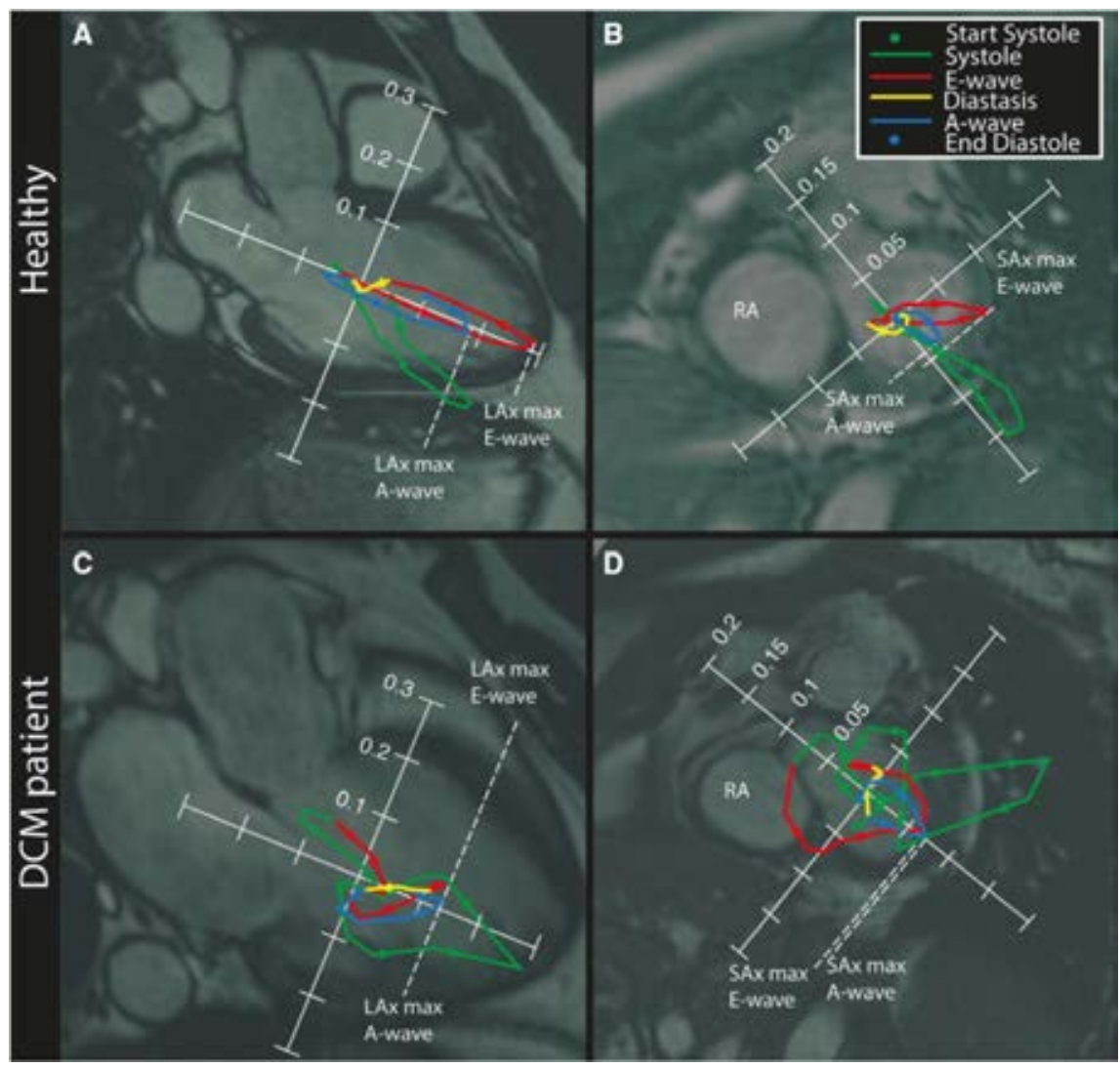

Figure 20: Hemodynamic forces over systole and diastole projected on a threechamber plane image (A and $\mathrm{C}$ ) and a short-axis plane image (B and D). Healthy subject (A and $\mathrm{B})$ and $\mathrm{DCM}$ patient $(\mathrm{C}$ and $\mathrm{D})$. Arrows along the curve indicate the direction of the force over time. (89) 
The SAx/LAx-ratio was in paper IV compared in a group of heart failure patients with LBBB to patients without LBBB but with similar severity of LV dilatation and dysfunction. The LV hemodynamic force at early diastolic LV filling is more directed towards the LV free wall (reflected as a higher SAx/LAx-ratio) in heart failure patients with LBBB compared to those without LBBB. No intergroup difference was observed during late diastolic filling. Moreover, the SAx/LAx-ratio and QRS duration correlated during early but not during late diastole. 


\section{DISCUSSION}

Studies included in this thesis have provided new insight into the complex nature of multidimensional blood flow within the cardiac ventricles by the use of novel 4D Flow CMR approaches.

\subsection{Physiological considerations}

$\mathrm{RV}$ function is a powerful prognostic indicator in many forms of heart disease. Assessment of the RV remains challenging and inexact, in part due to its complex shape. Using 4D flow CMR, the distribution, volume and energetics of different flow components could be investigated in normal RVs (paper I). Similar to that of the LV, diastolic flow through the normal RV appears to create favorable conditions for effective systolic ejection. Flow specific aspects of diastolic-systolic coupling described in paper I provide novel perspectives on LV and RV physiology and may add to the understanding of its pathophysiology.

The relatively short course of the Direct Flow through the RV and LV and the preservation of KE at ED may be an indication of efficient flow through the ventricular chambers. Rather than stop moving, the Direct Flow volume keeps some of its $\mathrm{KE}$ and is directed towards the outflow tract as late diastole transitions to systolic ejection. Such flow characteristics suggest that ventricular ejection of blood during systole is not only the product of myocardial contraction, but has already been prepared for ejection during diastole (60). Thus, effective LV ejection should be looked on as a product of effective coupling of diastolic inflow and preparation of blood volume with the ensuing systolic outflow, which previously has been proposed (92).

In paper III, altered flow patterns in heart failure patients with LBBB are associated with decreased preservation of KE during diastole which might be unfavorable for efficient ejection during systole and impaired diastolicsystolic coupling. This inefficient flow of blood through the heart may influence diastolic wall stress, and thus, contribute to further myocardial remodeling. Furthermore, the altered diastolic flow routes and impaired preservation of pre-systolic KE have been demonstrated in compensated heart failure patients with mildly remodeled LVs compared to normal LVs despite similar LV stroke volume (91). 
Blood flow through the heart is not a uniform entity going in and out of the heart every heartbeat, but rather a composition of many flow layers. A portion of the LV blood is always left in the ventricular chamber at the end of each systolic contraction. A feature of the intracardiac flow is that the functional limit may not only be defined by the borders of the endocardium, but also by the boundaries of different flow components. The distribution of the Residual Volume in the apical parts of the ventricles, observed in both RV and LV, may provide a fluid-fluid interaction that influence the route of inflowing blood in a way to promote smooth redirection and efficient systolic ejection.

The amount of Direct Flow KE preserved in a time frame at ED in the RV or the LV is small compared to the blood's total KE during the whole systole. Nevertheless, the preservation of KE might still be beneficial as loss of additional KE due to deceleration of blood would be converted to heat or pressure with an increase of diastolic pressure. Although this effect might be insignificant from one heartbeat to another, constant small loss of $\mathrm{KE}$ over countless of heartbeats may be disadvantageous for the heart over time.

TKE in normal LVs could be measured using 4D Flow CMR (paper II) and can address the prevailing uncertainty about whether there is any significant turbulence in healthy LVs. TKE values in normal LVs ranges from zero to approximately $5 \mathrm{~mJ}$, which is in line with previously assessed magnitude of TKE in the normal LA and ascending aorta.

An interesting aspect of intraventricular flow fields is the existence of diastolic vortices or vortex rings within the ventricles. In the normal RV during early diastolic filling, a vortex ring forms in the vicinity of tricuspid leaflets and moves into the RV. The superior portion of this vortex extends into the RV outflow tract and is attributed to the Direct Flow. The inferior part consist more of Retained Inflow and follows an inferior path towards the central RV, replacing blood already residing in the RV. During diastasis the vortex expands and its rotation velocity decreases before it accelerates following late diastolic filling. In normal LV, during early and late diastolic filling a vortex ring forms, originating from the distal tip of the MV leaflets (93). At late diastole, the vortex ring extends in the direction of the outflow tract. This rotating flow help redirect late inflow towards optimal systolic ejection. This corresponds to flow patterns in normal LVs which shows that a substantial portion of the late diastolic inflow volume passes smoothly to ensuing ejection. TKE values in normal LVs are also lower at late diastole than at early diastole. In dilated LVs of 
heart failure patients, a large vortex is occupying the center of the LV cavity. Most of the late inflowing volume in failing LVs is retained and progresses deeper into the LV and augments the early inflowing vortex in the center of the LV. This is in line with elevated TKE values observed in failing LVs located in the central part of the cavity.

Assessment of vortex formation in the cardiac ventricles has recently drawn attention as a new approach for evaluation of flow efficiency and cardiac function. Patient studies have shown that the incidence, location, and extent of vortex flow inside the LV were markedly altered in patients with dilated cardiomyopathy $(94,95)$. Delayed vortex formation during early diastolic filling and diminished vortex KE preservation was observed in heart failure patients with LBBB compared to healthy subjects using echocardiographic particle image velocimetry (96). This suggest that a relationship exists between abnormal vortex formation and LV dysfunction. However there is still no consensus on whether or not ventricular vortices have a beneficial role in terms of energy preservation.

Late diastolic TKE values are higher in DCM patients than in healthy subjects and this is associated with inflowing blood in the central part of the LV (paper II). These findings may result in irreversible conversion of kinetic energy into heat by viscous dissipation. Although, the effect on the total cardiac workload is most likely small, these KE losses can reflect inefficient intraventricular flow patterns.

LBBB causes a delay in early diastolic relaxation of the LV myocardium compared to the RV. In particular, the outward motion of the LV lateral wall is delayed. In studies on normal LV flow mentioned previously, early inflowing Direct Flow extends further into the LV than late inflowing Direct Flow, after which it redirects towards the outflow tract (64). Atrial contraction accelerates flow towards the LV outflow tract and may boost the velocity of early inflowing Direct Flow already residing close to the outflow tract. The reduced KE of early inflowing Direct Flow at ED observed in the presence of LBBB (paper III) may be due to the fact that the increase of flow velocity of early inflowing Direct Flow in late diastole is diminished as a consequence of a delayed inflow of early Direct Flow. $\mathrm{KE}$ at ED for the late inflowing Direct Flow was not different between the groups as late diastole is more dependent on atrial contraction which is less influenced by the delay in early diastolic relaxation of the LV myocardium.

In paper IV, there was no significant difference in the sphericity index between heart failure patients with and without LBBB. This suggests that 
the increased SAx/LAx-ratio, which is attributed to the early inflow, in LBBB patients is not caused by altered geometry of the dilated ventricles. As previously mentioned, LV relaxation is delayed in LBBB and especially the outward motion of the LV free wall. Furthermore, as RV diastolic filling phase occurs before LV diastolic filling, a transeptal pressure gradient can develop during early diastole which may allow the interventricular septum to bulge into the LV cavity. These mechanical distortions could influence early inflow patterns and be reflected as increased force in the SAx plane.

\subsection{Methodological considerations}

4D Flow CMR is a unique noninvasive technique enabling a comprehensive access of multidimensional blood flow through the human heart. Flow component visualization and quantification, and assessment of $\mathrm{KE}$, TKE and hemodynamic force are innovative 4D Flow CMR approaches used in this thesis to characterize different aspects of intraventricular flow.

Although the component analysis depends on observer variability, the assessment of the volumes of the LV flow components have showed both a high intra- and inter-observer reproducibility. TKE measurements with CMR have been compared to a gold-standard particle image velocimetry method and the results showed that there was a strong correlation between these two approaches.

In this thesis, the CMR data is acquired at a defined phase of the respiratory cycle, at rest and with the patient in supine body position. The data is therefore only representative under these conditions. As valvular disease was an exclusion criteria in the current papers, abnormal flow patterns related to valvular stenosis or regurgitation is not described. Different respiratory phases have an impact on cardiac blood flow, especially in the RV. Similarly, prone body position present a different preload to the ventricles. Recently, 4D Flow CMR acquisitions on patients with dobutamine infusion have been performed which can give insight into any flow-specific alternations occurring during increased heart rate and enhanced inotropy.

Artifacts such as phase wraps and background phase offsets can be detected by evaluation of 4D Flow CMR source images. Because 4D Flow CMR covers a volume and the flows within, the data can be analyzed for 
internal consistency by applying the principle of conservation of mass. Flow volume that enters and leaves the left or right ventricle during the analyzed heart cycle or a number of pathlines to and from a specific region of interest should be equal. A paper on validation of the flow component analysis showed accurate LV inflow and outflow volumes (61). During data visualization, data can further be checked for particle traces that suddenly change direction which can indicate velocity aliasing or phase wraps. Some streamlines or pathlines extend outside the lumen due to uncorrected background phase offset. Phase-unwrapping algorithms can be used to partially correct for aliasing (58). These steps can assure data quality.

Due to the fact that CMR data are collected during multiple heart cycles, temporary flow instabilities or beat-to-beat variation will be smoothed out. This limitation makes 4D Flow CMR insensitive to rapid or very small flow variations (46). However, global flow features that recur repeatedly throughout many cardiac cycles can be measured.

CMR images suffer from noise which is related to VENC and SNR. Noise is a source of error that can degrade the quality of phase-contrast velocity images. If pathlines are followed too long, they accumulate noise. In the flow analysis, instead of tracing pathlines over the whole cardiac cycle at once, pathlines are traced from ED forward and backward to the time of ES, thus reducing error. With higher magnetic field MRI, noise can be improved. Velocity-encoded data acquired at $3 \mathrm{~T}$ have improved SNR compared to $1.5 \mathrm{~T}(97,98)$. This benefit could allow further improvements in temporal and spatial resolution.

New development in CMR such as multidimensional parallel imaging that accelerates image acquisition, may address current limitation of $4 \mathrm{D}$ flow acquisition, mainly long scan times and limited spatial resolution (54). "The dual VENC" technique may in the future reduce aliasing artifacts.

\subsection{Clinical considerations}

4D Flow MRI can markedly improve the visualization and quantification of multidimensional blood flow in the heart which is not assessable by conventional echo Doppler assessments. Such new flow-specific insights into ventricular physiology (paper I) and pathophysiology (paper II-IV) have a potential clinical value. In abnormal cardiac states, 4D Flow MRI 
have the potential to identify early pathological changes that would otherwise not have been detected using other modalities.

Measures such as reduced Direct Flow volume and reduced Direct Flow $\mathrm{KE}$ may be used as markers of impaired cardiac function and aid in the assessment of systolic and/or diastolic dysfunction. Increased TKE observed in DCM patients in paper II may be used as a marker of diastolic dysfunction in clinically compensated heart failure patients. Reduced KE and increased TKE are aspects of inefficient flow of blood through the heart which may influence diastolic wall stress, and thus, contribute to further myocardial remodeling.

Cardiac remodeling is an important target in heart failure treatment. Many trials have showed that progression of heart failure may be delayed or even prevented by treatment of asymptomatic LV dysfunction (5). Accurate flow assessment has potential, in conjunction with traditional parameters based on wall motion and chamber dimensions, to be used in clinical decision-making and as a diagnostic tool. Further, 4D Flow MRI evaluation of intraventricular flow in heart failure patients may be used for monitoring treatment and identifying responders in order to optimize therapeutic strategies.

CRT is expensive and a significant proportion of heart failure patients undergoing this therapy do not demonstrate clinical improvement. Accordingly, there is a need for improved assessment of LV mechanical dyssynchrony for better identification of responders to CRT. Findings in paper III and paper IV suggest that 4D flow specific measures could serve as additional tools of LV mechanical dyssynchrony in heart failure patients, and could possibly be considered as potential useful predictors of response to CRT.

Recently, fully automatic segmentation and analysis of 4D Flow data have emerged which can speed up analysis and improve reproducibility (99). These are important factors that could make 4D Flow more available in clinical application. 


\section{ACKNOWLEDGEMENTS}

My research work in 4D Flow CMR began already in the beginning of 2010. It was at that time only a part of the obligatory independent scientific project during the sixth term of medical school. My interest and involvement in the research field grew successively thanks to the inspiration and the support of my supervisor and the personal at the Division of Cardiovascular Medicine and it was a great excitement when my first paper was published in 2011. I applied and was accepted to the doctoral research studies in the beginning of 2012 and since then they have proceeded parallel to my medical studies in Linköping and the internship/clinical carrier in Stockholm. Managing both clinical work and research studies has been challenging and sometimes even exhausting. It would not have been possible without the encouragement and devotion of my supervisor Carl-Johan Carlhäll, to whom I would like to express my deepest thanks. You have truly been the backbone to me as an aspiring researcher and clinical doctor.

The 4D Flow CMR group was fairly small back in 2010 and I would first like to acknowledge those that have been there from the start, bringing major contribution to the research field as well as to my research studies: my co-supervisor Tino Ebbers for many inspiring discussions and very valuable advises; my other co-supervisor Petter Dyverfeldt for the energy of putting up with all of my stupid questions and still being able to answer all of them; Jonathan Eriksson for always finding solutions to my analysis errors and providing me with technical tools; Ann F. Bolger for practically always being available (all the way from California, USA) and bringing spot-on comments and linguistic expertise; and Alexandru G. Fredriksson for the great work in collaborating the important first paper. Thank you, Jan Engwall and Eva Nylander for being my role models as clinical doctors and for providing invaluable cardiovascular knowledge. Thank to Sven Petersson, Henrik Haraldsson and Andreas Sigfridsson for good input, nice company and discussions, both research and nonresearch related - I wish you all the best in your new exciting endeavors.

Since 2010 the division has grown more and more with new colleagues joining the team, bringing new fresh ideas. Thank you Magnus Ziegler for finding solution to some of my most troubling problems (and for all the great bike rides) and Federica Viola for developing and simplifying my most important tool (and for the Italian cuisine). Thanks also to my other colleagues at the institution for great support: Jonas Lantz, Sofia Kvernby, Mariana Bustamante, Belén Casas, Merih Cibis, Vikas 
Gupta and Hojin Ha. I am not the only medical doctor at the institution who simultaneously juggled with clinical and doctoral duties - thanks to Kristofer Hedman and Lena Forsberg for tips and tricks to manage it all through.

I would like to extend my acknowledgements to Johan Kihlberg for skillful acquisition of the CMR data, to Urban Alehagen for recruiting many of the patients in my studies and to Malin Strand for always helping me with intricate administrative troubles. Thanks to everyone at the Department of Clinical Physiology for great cooperation.

Thanks to all of my close friends who have given me lots of joy over the years and for cheering on me.

Lastly and certainly not least, I would like to acknowledge my family: my mother Józefa and father Stanislaw, both research works, for always being there and tirelessly helping me conduct my studies, even when it meant traveling numerous times back and forth between Stockholm and Linköping; my brother Pawel for being the greatest inspiration in the world (who got his own $\mathrm{PhD}$ when I just started my medical studies) and his family Helena and Majken for your support; my beloved fiancée Maria for your never-ending love and understanding, especially during the last intensive part of my doctorate - I long for moving with you to our new apartment and beginning the next chapter of our lives together.

Jakub Zajac

August 2017 


\section{REFERENCES}

1. Braunwald E, Bonow RO: Braunwald's Heart Disease : A Textbook of Cardiovascular Medicine. Saunders; 2012.

2. Brignole M, Auricchio A, Baron-Esquivias G, et al.: 2013 ESC Guidelines on cardiac pacing and cardiac resynchronization therapy: the Task Force on cardiac pacing and resynchronization therapy of the European Society of Cardiology (ESC). Developed in collaboration with the European Heart Rhythm Association (EHRA). Eur Heart $J$ 2013; 34:2281-329.

3. Stewart S, MacIntyre K, Hole DJ, Capewell S, McMurray JJ: More "malignant" than cancer? Five-year survival following a first admission for heart failure. Eur J Heart Fail 2001; 3:315-22.

4. McMurray JJ V, Pfeffer MA: Heart failure. Lancet 2005:1877-1889.

5. Ponikowski P, Voors AA, Anker SD, et al.: 2016 ESC Guidelines for the diagnosis and treatment of acute and chronic heart failure. Eur Heart $J$ 2016:2129-2200m.

6. Mann DL, Bristow MR: Mechanisms and models in heart failure: The biomechanical model and beyond. Circulation 2005:2837-2849.

7. Hove JR, Köster RW, Forouhar AS, Acevedo-Bolton G, Fraser SE, Gharib M: Intracardiac fluid forces are an essential epigenetic factor for embryonic cardiogenesis. Nature 2003; 421:172-7.

8. Boron WF, Boulpaep EL: Medical Physiology. .

9. Cheng CP, Igarashi Y, Little WC: Mechanism of augmented rate of left ventricular filling during exercise. Circ Res 1992; 70:9-19.

10. Alhogbani T, Strohm O, Friedrich MG: Evaluation of left atrial contraction contribution to left ventricular filling using cardiovascular magnetic resonance. J Magn Reson Imaging 2013; 37:860-864.

11. Ho SY: Anatomy and myoarchitecture of the left ventricular wall in normal and in disease. Eur J Echocardiogr 2009; 10:iii3-7.

12. Nielsen E, Smerup M, Agger P, et al.: Normal Right Ventricular ThreeDimensional Architecture, as Assessed with Diffusion Tensor Magnetic Resonance Imaging, is Preserved During Experimentally Induced Right Ventricular Hypertrophy. Anat Rec Adv Integr Anat Evol Biol 2009; 292:640-651.

13. Papadacci C, Finel V, Provost J, et al.: Imaging the dynamics of cardiac fiber orientation in vivo using 3D Ultrasound Backscatter Tensor Imaging. Sci Rep 2017; 7:830.

14. Santamore WP, Constantinescu M, Vinten-Johansen J, Johnston WE, Little WC: Alterations in left ventricular compliance due to changes in right ventricular volume, pressure and compliance. Cardiovasc Res 1988; 22:768-76. 
15. Santamore WP, Lynch PR, Heckman JL, Bove AA, Meier GD: Left ventricular effects on right ventricular developed pressure. J Appl Physiol $1976 ; 41$.

16. Mann DL: Basic mechanisms of left ventricular remodeling: the contribution of wall stress. J Card Fail 2004; 10(6 Supp1):S202-6.

17. Floras JS: Sympathetic Nervous System Activation in Human Heart Failure. Clinical Implications of an Updated Model. J Am Coll Cardiol 2009:375-385.

18. Guang C, Phillips RD, Jiang B, Milani F: Three key proteases Angiotensin-I-converting enzyme (ACE), ACE2 and renin - Within and beyond the renin-angiotensin system. Arch Cardiovasc Dis 2012:373-385. 19. Shafiq M, Miller AB: Blocking aldosterone in heart failure. Ther $A d v$ Cardiovasc Dis 2009; 3:379-385.

20. Korinek J, Boerrigter G, Mohammed SF, Burnett JC: Insights into natriuretic peptides in heart failure: An update. Curr Heart Fail Rep 2008:97-104.

21. Carnicer R, Crabtree MJ, Sivakumaran V, Casadei B, Kass DA: Nitric oxide synthases in heart failure. Antioxid Redox Signal 2013; 18:10781099.

22. Kumar V, Abbas AK, Aster JC, Robbins SL (Stanley L: Robbins Basic Pathology. .

23. Opie LH, Commerford PJ, Gersh BJ, Pfeffer MA: Controversies in ventricular remodelling. Lancet (London, England) 2006; 367:356-67.

24. Sabbah HN, Goldstein S: Ventricular remodelling: consequences and therapy. Eur Heart J 1993; 14 Suppl C:24-9.

25. Movassagh M, Choy MK, Knowles DA, et al.: Distinct epigenomic features in end-stage failing human hearts. Circulation 2011; 124:24112422.

26. Misra A, Mann DL: Treatment of heart failure beyond practice guidelines. Role of cardiac remodeling. Circ J 2008; 72 Suppl A:A1-7.

27. Linzbach AJ: Heart failure from the point of view of quantitative anatomy. Am J Cardiol 1960; 5:370-382.

28. Ross J: Mechanisms of cardiac contraction. What roles for preload, afterload and inotropic state in heart failure? Eur Heart $J$ 1983; 4 Suppl A:19-28.

29. Vasan RS: Diastolic heart failure. BMJ 2003; 327:1181-2.

30. Packer M, Bristow M, Cohn J, et al.: The effect of carvedilol on morbidity and mortality in patients with chronic heart failure. $N$ Engl $J$ Med 1996; 334:1349-1355.

31. Bristow MR, Gilbert EM, Abraham WT, et al.: Carvedilol produces dose-related improvements in left ventricular function and survival in subjects with chronic heart failure. MOCHA Investigators. Circulation 
1996; 94:2807-2816.

32. SOLVD T, Investigattors, Yusuf $\mathrm{S}$, et al.: Effect of enalapril on mortality and the development of heart failure in asymptomatic patients with reduced left ventricular ejection fractions. The SOLVD Investigattors.[Erratum appears in N Engl J Med 1992 Dec 10;327(24):1768]. N Engl J Med 1992; 327:685-691.

33. Cohn JN, Johnson G, Ziesche S, et al.: A comparison of enalapril with hydralazine-isosorbide dinitrate in the treatment of chronic congestive heart failure. N Engl J Med 1991; 325:303-310.

34. Mann DL, Barger PM, Burkhoff D: Myocardial recovery and the failing heart: Myth, magic, or molecular target? J Am Coll Cardiol 2012:2465-2472.

35. Linde C, Ellenbogen K, McAlister FA: Cardiac resynchronization therapy (CRT): Clinical trials, guidelines, and target populations. Hear Rhythm 2012; 9:S3-S13.

36. Weeks KL, McMullen JR: The athlete's heart vs. the failing heart: can signaling explain the two distinct outcomes? Physiology (Bethesda) 2011; 26:97-105.

37. Scharhag J, Schneider G, Urhausen A, Rochette V, Kramann B, Kindermann W: Athlete's heart: right and left ventricular mass and function in male endurance athletes and untrained individuals determined by magnetic resonance imaging. J Am Coll Cardiol 2002; 40:1856-1863. 38. Shephard RJ, Balady GJ: Exercise as cardiovascular therapy. Circulation 1999; 99:963-72.

39. Ellison GM, Waring CD, Vicinanza C, Torella D: Physiological cardiac remodelling in response to endurance exercise training: cellular and molecular mechanisms. Heart 2012; 98:5-10.

40. Kheradvar A, Pedrizzetti G: Fundamental Fluid Mechanics. In Vor Form Cardiovasc Syst. London: Springer London; 2012:1-16.

41. Yonus A. Engel JMC: Fluid Mechanics Fundamentals and Applications. McGraw-Hill; 2006.

42. Kim WY, Walker PG, Pedersen EM, et al.: Left ventricular blood flow patterns in normal subjects: A quantitative analysis by three-dimensional magnetic resonance velocity mapping. J Am Coll Cardiol 1995; 26:224238.

43. Richter Y, Edelman ER: Cardiology Is Flow. Circulation 2006; 113:2679-2682.

44. Cheng C, Tempel D, Van Haperen R, et al.: Atherosclerotic lesion size and vulnerability are determined by patterns of fluid shear stress. Circulation 2006; 113:2744-2753.

45. Yang G-Z, Merrifield R, Masood S, Kilner PJ: Flow and myocardial interaction: an imaging perspective. Philos Trans R Soc Lond B Biol Sci 
2007; 362:1329-1341.

46. Carlhall CJ, Bolger A: Passing Strange: Flow in the Failing Ventricle. Circ Hear Fail 2010; 3:326-331.

47. Kilner PJ, Yang G-Z, Wilkes AJ, Mohiaddin RH, Firmin DN, Yacoub MH: Asymmetric redirection of flow through the heart. Nature 2000; 404:759-761.

48. Jacobs LE, Kotler MN, Parry WR: Flow patterns in dilated cardiomyopathy: a pulsed-wave and color flow Doppler study. J Am Soc Echocardiogr ; 3:294-302.

49. Rodevand O, Bjornerheim R, Edvardsen T, Smiseth OA, Ihlen H: Diastolic flow pattern in the normal left ventricle. J Am Soc Echocardiogr 1999; 12:500-7.

50. Kwong RY: Cardiovascular Magnetic Resonance Imaging. Humana; 2008.

51. Hundley WG, Bluemke DA, Finn JP, et al.: ACCF/ACR/AHA/NASCI/SCMR 2010 Expert Consensus Document on Cardiovascular Magnetic Resonance. J Am Coll Cardiol 2010; 55:26142662.

52. Srichai MB, Lim RP, Wong S, Lee VS: Cardiovascular applications of phase-contrast MRI. AJR Am J Roentgenol 2009; 192:662-75.

53. Chai P, Mohiaddin R: How we perform cardiovascular magnetic resonance flow assessment using phase-contrast velocity mapping. $J$ Cardiovasc Magn Reson 2005; 7:705-16.

54. Markl M, Frydrychowicz A, Kozerke S, Hope M, Wieben O: 4D flow MRI. J Magn Reson Imaging 2012; 36:1015-1036.

55. Bernstein MA, Shimakawa A, Pelc NJ: Minimizing TE in momentnulled or flow-encoded two- and three-dimensional gradient-echo imaging. J Magn Reson Imaging ; 2:583-8.

56. Ebbers T: Flow Imaging: Cardiac Applications of 3D Cine PhaseContrast MRI. Curr Cardiovasc Imaging Rep 2011:127-133.

57. Mark1 M, Kilner PJ, Ebbers T: Comprehensive 4D velocity mapping of the heart and great vessels by cardiovascular magnetic resonance. $J$ Cardiovasc Magn Reson 2011; 13:7.

58. Dyverfeldt P, Bissell M, Barker AJ, et al.: 4D flow cardiovascular magnetic resonance consensus statement. J Cardiovasc Magn Reson 2015; 17:72.

59. Wigström L, Sjöqvist L, Wranne B: Temporally resolved 3D phasecontrast imaging. Magn Reson Med 1996; 36:800-3.

60. Fredriksson AG, Zajac J, Eriksson J, et al.: 4-D blood flow in the human right ventricle. Am J Physiol Heart Circ Physiol 2011; 301:H234450.

61. Eriksson J, Carlhäll CJ, Dyverfeldt P, Engvall J, Bolger AF, Ebbers T: 
Semi-automatic quantification of 4D left ventricular blood flow. $J$ Cardiovasc Magn Reson 2010; 12:9.

62. Eriksson J, Bolger AF, Ebbers T, Carlhäll C-J: Four-dimensional blood flow-specific markers of LV dysfunction in dilated cardiomyopathy. Eur Heart J Cardiovasc Imaging 2013; 14:417-24.

63. Bolger AF, Heiberg E, Karlsson M, et al.: Transit of blood flow through the human left ventricle mapped by cardiovascular magnetic resonance. $J$ Cardiovasc Magn Reson 2007; 9:741-7.

64. Eriksson J, Dyverfeldt P, Engvall J, Bolger AF, Ebbers T, Carlhäll CJ: Quantification of presystolic blood flow organization and energetics in the human left ventricle. Am J Physiol Heart Circ Physiol 2011; 300:H213541.

65. Dyverfeldt P, Kvitting J-PE, Sigfridsson A, Engvall J, Bolger AF, Ebbers T: Assessment of fluctuating velocities in disturbed cardiovascular blood flow: in vivo feasibility of generalized phase-contrast MRI. J Magn Reson Imaging 2008; 28:655-63.

66. Wigström L, Ebbers T, Fyrenius A, et al.: Particle trace visualization of intracardiac flow using time-resolved 3D phase contrast MRI. Magn Reson Med 1999; 41:793-9.

67. Töger J, Carlsson M, Söderlind G, et al.: Volume Tracking: A new method for quantitative assessment and visualization of intracardiac blood flow from three-dimensional, time-resolved, three-component magnetic resonance velocity mapping. BMC Med Imaging 2011; 11:10.

68. Fluckiger JU, Goldberger JJ, Lee DC, et al.: Left atrial flow velocity distribution and flow coherence using four-dimensional FLOW MRI: a pilot study investigating the impact of age and Pre- and Postintervention atrial fibrillation on atrial hemodynamics. J Magn Reson Imaging 2013; 38:580-7.

69. Fyrenius A, Wigström L, Ebbers T, Karlsson M, Engvall J, Bolger AF: Three dimensional flow in the human left atrium. Heart 2001; 86:448-55. 70. Kvitting J-PE, Dyverfeldt P, Sigfridsson A, et al.: In vitro assessment of flow patterns and turbulence intensity in prosthetic heart valves using generalized phase-contrast MRI. J Magn Reson Imaging 2010; 31:107580.

71. Westenberg JJM, Roes SD, Ajmone Marsan N, et al.: Mitral valve and tricuspid valve blood flow: accurate quantification with 3D velocityencoded MR imaging with retrospective valve tracking. Radiology 2008; 249:792-800.

72. Kozerke S, Hasenkam JM, Pedersen EM, Boesiger P: Visualization of flow patterns distal to aortic valve prostheses in humans using a fast approach for cine 3D velocity mapping. J Magn Reson Imaging 2001; 13:690-8. 
73. Dyverfeldt P, Hope MD, Tseng EE, Saloner D: Magnetic resonance measurement of turbulent kinetic energy for the estimation of irreversible pressure loss in aortic stenosis. JACC Cardiovasc Imaging 2013; 6:64-71. 74. Frydrychowicz A, Markl M, Hirtler D, et al.: Aortic hemodynamics in patients with and without repair of aortic coarctation: in vivo analysis by 4D flow-sensitive magnetic resonance imaging. Invest Radiol 2011; 46:317-25.

75. Markl M, Draney MT, Miller DC, et al.: Time-resolved threedimensional magnetic resonance velocity mapping of aortic flow in healthy volunteers and patients after valve-sparing aortic root replacement. $J$ Thorac Cardiovasc Surg 2005; 130:456-63.

76. Kvitting J-PE, Ebbers T, Wigström L, Engvall J, Olin CL, Bolger AF: Flow patterns in the aortic root and the aorta studied with time-resolved, 3-dimensional, phase-contrast magnetic resonance imaging: implications for aortic valve-sparing surgery. J Thorac Cardiovasc Surg 2004; 127:1602-7.

77. Hope MD, Hope TA, Crook SES, et al.: 4D flow CMR in assessment of valve-related ascending aortic disease. JACC Cardiovasc Imaging 2011; 4:781-7.

78. Reiter G, Reiter U, Kovacs G, et al.: Magnetic resonance-derived 3dimensional blood flow patterns in the main pulmonary artery as a marker of pulmonary hypertension and a measure of elevated mean pulmonary arterial pressure. Circ Cardiovasc Imaging 2008; 1:23-30.

79. Markl M, Wegent F, Zech T, et al.: In vivo wall shear stress distribution in the carotid artery: effect of bifurcation geometry, internal carotid artery stenosis, and recanalization therapy. Circ Cardiovasc Imaging 2010; 3:647-55.

80. Bammer R, Hope TA, Aksoy M, Alley MT: Time-resolved 3D quantitative flow MRI of the major intracranial vessels: initial experience and comparative evaluation at $1.5 \mathrm{~T}$ and $3.0 \mathrm{~T}$ in combination with parallel imaging. Magn Reson Med 2007; 57:127-40.

81. Binter C, Knobloch V, Manka R, Sigfridsson A, Kozerke S: Bayesian multipoint velocity encoding for concurrent flow and turbulence mapping. Magn Reson Med 2013; 69:1337-45.

82. Dyverfeldt P, Sigfridsson A, Kvitting J-PE, Ebbers T: Quantification of intravoxel velocity standard deviation and turbulence intensity by generalizing phase-contrast MRI. Magn Reson Med 2006; 56:850-8.

83. Zajac J, Eriksson J, Dyverfeldt P, Bolger AF, Ebbers T, Carlhäll C-J: Turbulent kinetic energy in normal and myopathic left ventricles. J Magn Reson Imaging 2015; 41:1021-9.

84. Tyszka JM, Laidlaw DH, Asa JW, Silverman JM: Three-dimensional, time-resolved (4D) relative pressure mapping using magnetic resonance 
imaging. J Magn Reson Imaging 2000; 12:321-9.

85. Ebbers T, Wigström L, Bolger AF, Wranne B, Karlsson M: Noninvasive measurement of time-varying three-dimensional relative pressure fields within the human heart. J Biomech Eng 2002; 124:288-93. 86. Eriksson J, Bolger AF, Carlhäll C-J, Ebbers T: Spatial heterogeneity of four-dimensional relative pressure fields in the human left ventricle. Magn Reson Med 2015; 74:1716-25.

87. Oyre S, Paaske WP, Ringgaard S, et al.: Automatic accurate noninvasive quantitation of blood flow, cross-sectional vessel area, and wall shear stress by modelling of magnetic resonance velocity data. Eur J Vasc Endovasc Surg 1998; 16:517-24.

88. Frydrychowicz A, Stalder AF, Russe MF, et al.: Three-dimensional analysis of segmental wall shear stress in the aorta by flow-sensitive fourdimensional-MRI. J Magn Reson Imaging 2009; 30:77-84.

89. Eriksson J, Bolger AF, Ebbers T, Carlhäll C-J: Assessment of left ventricular hemodynamic forces in healthy subjects and patients with dilated cardiomyopathy using 4D flow MRI. Physiol Rep 2016; 4:e12685. 90. Mohiaddin RH: Flow patterns in the dilated ischemic left ventricle studied by MR imaging with velocity vector mapping. J Magn Reson Imaging 1995; 5:493-498.

91. Svalbring E, Fredriksson A, Eriksson J, et al.: Altered Diastolic Flow Patterns and Kinetic Energy in Subtle Left Ventricular Remodeling and Dysfunction Detected by 4D Flow MRI. PLoS One 2016; 11:e0161391.

92. Bolger AF, Heiberg E, Karlsson M, et al.: Transit of blood flow through the human left ventricle mapped by cardiovascular magnetic resonance. $J$ Cardiovasc Magn Reson 2007; 9:741-7.

93. Elbaz MSM, Calkoen EE, Westenberg JJM, Lelieveldt BPF, Roest AAW, van der Geest RJ: Vortex flow during early and late left ventricular filling in normal subjects: quantitative characterization using retrospectively-gated 4D flow cardiovascular magnetic resonance and three-dimensional vortex core analysis. J Cardiovasc Magn Reson 2014; 16:78.

94. Rodriguez Muñoz D, Mark1 M, Moya Mur JL, et al.: Intracardiac flow visualization: current status and future directions. Eur Heart J Cardiovasc Imaging 2013; 14:1029-38.

95. Hong G-R, Pedrizzetti G, Tonti G, et al.: Characterization and Quantification of Vortex Flow in the Human Left Ventricle by Contrast Echocardiography Using Vector Particle Image Velocimetry. JACC Cardiovasc Imaging 2008; 1:705-717.

96. Gürel E, Prinz C, Van Casteren L, Gao H, Willems R, Voigt J-U: The Impact of Function-Flow Interaction on Left Ventricular Efficiency in Patients with Conduction Abnormalities: A Particle Image Velocimetry 
and Tissue Doppler Study. J Am Soc Echocardiogr 2016; 29:431-40.

97. Lotz J, Döker R, Noeske R, et al.: In vitro validation of phase-contrast flow measurements at $3 \mathrm{~T}$ in comparison to $1.5 \mathrm{~T}$ : Precision, accuracy, and signal-to-noise ratios. J Magn Reson Imaging 2005; 21:604-610.

98. Bock J, Frydrychowicz A, Stalder AF, et al.: 4D phase contrast MRI at 3 T: Effect of standard and blood-pool contrast agents on SNR, PC-MRA, and blood flow visualization. Magn Reson Med 2010; 63:330-338.

99. Bustamante M, Petersson S, Eriksson J, et al.: Atlas-based analysis of 4D flow CMR: Automated vessel segmentation and flow quantification. $J$ Cardiovasc Magn Reson 2015; 17:87. 


\section{Papers}

The articles associated with this thesis have been removed for copyright reasons. For more details about these see:

http://urn.kb.se/resolve?urn=urn:nbn:se:liu:diva-141006 